\title{
Cross-screening in observational studies that test many hypotheses
} Qingyuan Zhao, Dylan S. Small, Paul R. Rosenbaum 1

University of Pennsylvania, Philadelphia

Abstract. We discuss observational studies that test many causal hypotheses, either hypotheses about many outcomes or many treatments. To be credible an observational study that tests many causal hypotheses must demonstrate that its conclusions are neither artifacts of multiple testing nor of small biases from nonrandom treatment assignment. In a sense that needs to be defined carefully, hidden within a sensitivity analysis for nonrandom assignment is an enormous correction for multiple testing: in the absence of bias, it is extremely improbable that multiple testing alone would create an association insensitive to moderate biases. We propose a new strategy called "cross-screening," different from but motivated by recent work of Bogomolov and Heller on replicability. Cross-screening splits the data in half at random, uses the first half to plan a study carried out on the second half, then uses the second half to plan a study carried out on the first half, and reports the more favorable conclusions of the two studies correcting using the Bonferroni inequality for having done two studies. If the two studies happen to concur, then they achieve Bogomolov-Heller replicability; however, importantly, replicability is not required for strong control of the family-wise error rate, and either study alone suffices for firm conclusions. In randomized studies with a few hypotheses, cross-split screening is not an attractive method when compared with conventional methods of multiplicity control, but it can become attractive when hundreds or thousands of hypotheses are subjected to sensitivity analyses in an observational study. We illustrate the technique by comparing 46 biomarkers in individuals who consume large quantities of fish versus little or no fish.

Keywords: Bonferroni inequality; causal inference; design sensitivity; observational study; replicability; sample splitting; sensitivity analysis.

\footnotetext{
${ }^{1}$ Address for correspondence: Department of Statistics, The Wharton School, University of Pennsylvania, Jon M. Huntsman Hall, 3730 Walnut Street, Philadelphia, PA 19104-6340 USA. E-mail: dsmall@wharton.upenn.edu. 25 February 2017.
} 


\section{Introduction: testing many hypotheses in observational studies}

\subsection{Sensitivity analyses and corrections for multiple testing affect one another}

To be credible, an observational or nonrandomized study that tests many null hypotheses about treatment effects must demonstrate that claimed effects are neither an artifact of testing many hypotheses nor a consequence of small departures from randomized treatment assignment. These two demonstrations are related. Tiny treatment effects have almost no chance of being judged insensitive to small unmeasured biases from nonrandom assignment; see Rosenbaum $(2015, \S 5)$. With many null hypotheses, tiny treatment effects also have almost no chance of being distinguished from artifacts of multiple testing in studies of moderate sample size. These two considerations motivate screening to eliminate null or negligible treatment effects before testing, thereby greatly reducing the correction for multiple testing.

To see that tiny effects have almost no chance of being distinguished from artifacts of multiple testing in studies of moderate sample size, suppose there are $I$ independent observations on each of $K$ independent outcomes, where outcome $k$ is $N\left(\tau_{k}, 1\right)$, with the $k$ th null hypothesis asserting $H_{k}: \tau_{k}=0$, where in fact $\tau_{1}=\tau>0$ and $\tau_{2}=\tau_{3}=\cdots=\tau_{K}=0$. In this case, the probability that the only active group, $k=1$, has a sample mean above the $K-1$ other group means is $\varpi_{\tau, K, I}=\int_{-\infty}^{\infty} \Phi(y / \sqrt{I})^{K-1} \phi\{(y-\tau) / \sqrt{I}\} / \sqrt{I} d y$, where $\Phi(\cdot)$ and $\phi(\cdot)$ are, respectively, the standard Normal cumulative and density functions. With $I=100$ observations and $K=100$ hypotheses, $\varpi_{\tau, K, I}$ is 0.082 for $\tau=0.1,0.501$ for $\tau=0.25$ and 0.988 for $\tau=0.5$. So, we are unlikely to locate the one real effect if $\tau=0.1$ and very likely to locate it if $\tau=0.5$. As $I \rightarrow \infty$ with $\tau$ and $K$ fixed, $\varpi_{\tau, K, I} \rightarrow 1$; however, this limiting calculation is less relevant in the problems we often face in which $K$ is fairly large and $I$ is not enormous relative to $K$. An effect of $\tau=0.5$ with $I=100$ is also likely to be judged insensitive to substantial biases from nonrandom treatment assignment; see Rosenbaum (2010, §14.2, p. 268). As will be seen in later sections, we can reduce the magnitude of a correction for multiple testing by screening out negligible effects that never had a chance of surviving a sensitivity analysis, and had little chance of surviving a correction for multiple testing.

In the current paper, we propose a new technique called cross-screening. The sample is split in half at random. The first half is used to plan the analysis of the second half, for instance, selecting null hypotheses that appear to be false, the one-tailed direction of 
the likely departure from the null, and the best test statistic to use in testing. In parallel, the second half is used to plan the analysis of the first half. Both halves are analyzed using these data-derived plans, and the more favorable results of the two analyses are reported with a Bonferroni correction for performing two analyses. Both halves are used to plan and both halves are used to test, so cross-screening uses all of the data to test hypotheses. Cross-screening strongly controls the family-wise error rate, as discussed in 2.1. We compare cross-screening to alternative methods, such as a Bonferroni correction for testing $K$ two-sided hypotheses, or screening using a small fraction of the data. Crossscreening performs poorly except when $K$ is large and $I$ is not extremely large, but it often wins decisively in sensitivity analyses with large $K$ and moderate $I / K$. Cross-screening is related to, though distinct from, a concept of replicability developed by Bogomolov and Heller (2013); see $₫ 2.4$ and $₫ 6$ for detailed discussion. Cross-screening rejects hypothesis $H_{k}$ if either half-sample rejects $H_{k}$, thereby strongly controlling the family-wise rate; however, it achieves Bogomolov-Heller replicability if both halves reject $H_{k}$.

\subsection{Outline: a general method; measures of performance}

As it turns out, our simplest results are also our most definite and most useful results, so we present them first $₫ 2$, after fixing ideas with a motivating example in $\$ 1.3$. Section 4 presents a series of incomplete evaluations of cross-screening; however, the simulation in $\$ 5$ can be read immediately after $\oint_{2}$. Alas, the attraction of cross-screening is its flexibility, but it is this very flexibility that makes it difficult to offer a definitive evaluation. Suffice it to say here that cross-screening performs well in certain situations and from certain vantage points, but it performs poorly in others, and a user of the method needs to be aware of both aspects. The formal evaluation in $\$ 4$ requires some notation and definitions that are reviewed in $\$ 3$, whereas some of these same ideas appear informally in \$2, Finally, $\$ 6$ considers nonrandom cross-screening.

\subsection{Example: eating fish and biomarkers}

Using the National Health and Nutrition Examination Survey (NHANES) 2013-2014, we defined high fish consumption as more than 12 servings of fish or shellfish in the previous month, and low fish consumption as 0 or 1 servings of fish. We matched 234 adults with high fish consumption to 234 adults with low fish consumption, matching for gender, age, income, race, education and smoking. Income was measured as the ratio of income to the 
poverty level capped at five times poverty. Smoking was measured by two variables: (i) having smoked a total of at least 100 cigarettes, and (ii) cigarettes currently smoked per month. Figure 1 shows the distribution of age, income, education and smoking for the matched high and low groups. For brevity, we refer to high fish consumption as treated, and to low fish consumption as control.

We compared the treated and control groups in terms of the logs of $K=46$ biomarkers. All biomarkers were nonnegative, and when a biomarker could equal zero, we added one to that biomarker so that logs could be taken. Base 2 logs are used, so that a treated-minuscontrol difference of 1 implies the treated person's response is twice the matched control's response. Generally, if the difference is $y>0$ then the control's response must be doubled $y$ times to yield the matched treated subject's response, and if the the difference is $y<0$ then the treated subject's response must be doubled $y$ times to yield the matched control's response. Figure 2 shows the 234 treated-minus-control matched pair differences, $i=1,2$, $\ldots, I=234$, in the logs of the $K=46$ biomarkers, $k=1, \ldots, K=46$. A few biomarkers show large differences.

Table 1 compares two multiplicity-corrected sensitivity analyses for the data depicted in Figure 2. Method B applies a Bonferroni correction to Wilcoxon's signed rank test using the sensitivity analysis that is discussed in Rosenbaum $(1987 ; 2002, \S 4 ; 2011)$ and that is reviewed briefly in 93.2 . Specifically, method B performs $2 \times 46$ one-sided sensitivity analyses, one in each tail for $K=46$ outcomes, and obtains an upper bound on each onesided $P$-value in the presence of a bias of at most $\Gamma$. Here, $\Gamma \geq 1$ measures the magnitude of the departure from random assignment within pairs, such that two matched people might differ in their odds of treatment by a factor of $\Gamma$ due to some unmatched covariate; see 33.2 . The B method takes as the adjusted $P$-value for a biomarker the smaller of 1 and $2 \times K=2 \times 46$ times the smaller of the two $P$-value bounds for that biomarker. For $\Gamma=1$, this is simply the Bonferroni correction applied to $K=46$ two-sided Wilcoxon tests, and for $(\Gamma=1, K=1)$ it is simply a two-sided Wilcoxon test. For biomarkers $k=15$, 18,21 , and 23 , the corrected $P$-values are below 0.05 in a randomization test with $\Gamma=1$. Biomarker $k=15$ is sensitive to a nontrivial bias of $\Gamma=1.25$, but biomarkers $k=18,21$, and 23 are not. Biomarker $k=21$ becomes sensitive at $\Gamma=1.76$ with a corrected $P$-value bound of 0.054. Biomarkers $k=18$ and 23 are sensitive to a bias of $\Gamma=9$ with $P$-value bounds of 0.095 and 0.075 , respectively, but they are insensitive to a bias of $\Gamma=8$ with $P$-value bounds of 0.030 and 0.023 (not shown in Table 1). Perhaps unsurprisingly, the noticeable differences between high and low consumers of fish are high levels of mercury in 
the blood of high consumers.

When used in sensitivity analyses, the two-sided Bonferroni method is somewhat conservative. A brief explanation of this follows, but the remainder of this paragraph is not essential to the paper and may be skipped. It is correct but conservative to say: If the bias in treatment assignment is at most $\Gamma$, and if $K$ hypotheses are tested, then the probability is at most $\alpha$ that at least one true null hypothesis yields a one-sided $P$-value bound less than or equal to $\alpha /(2 K)$; see Rosenbaum and Silber (2009a, §4.5). The Bonferroni method is conservative for three reasons. First, of course, the Bonferroni inequality is an inequality, not an equality. Second, the one-sided $P$-value bound is obtained by maximizing the probability in one tail, thereby depleting the opposite tail, so doubling the one-sided $P$-value bound is conservative for a two-sided test when $\Gamma>1$. Third, when $\Gamma>1$, the worst pattern of biases for one outcome is often not the worst pattern for other outcomes, and the Bonferroni method ignores this. The Bonferroni-Holm method is least conservative when different outcomes have effects of very different magnitudes, and it is most conservative when many weakly correlated outcomes have effects of nearly the same size. Fogarty and Small (2016) propose an optimization technique that eliminates this third source of conservatism. An additional consideration, not strictly a form of conservatism, is that it may not be best to think about $K=46$ individual outcomes; rather, there may be greatest insensitivity to bias for some linear combination of the $K$ outcomes; see Rosenbaum (2016).

The second method in Table 1 is cross-screening (CS), and it is defined in 92 , Notably in Table 1, cross-screening judges outcomes $k=18$ and 23 to be insensitive to a bias of $\Gamma=9$ while method B judges them sensitive to $\Gamma=9$; indeed, method CS judges these two outcomes insensitive to a bias of $\Gamma=11$. As discussed later, we expect cross-screening to lose to method $\mathrm{B}$ in a randomization test, $\Gamma=1$, or when $I / K$ is large. We offer reasons to expect cross-screening to perform well, as it does in Table 1, when allowance is made for a nontrivial bias, $\Gamma \geq 1.25$, and $I / K$ is not large.

\section{What is random cross-screening?}

\subsection{Definition and control of the family-wise error rate}

Random cross-screening begins by splitting the data set in half at random, yielding two groups of $I / 2$ pairs, or $234 / 2=117$ pairs in Figure 2. The unusual aspect of cross-screening is that both halves are used to screen and both halves are used to test. Indeed, each half 
suggests a way to test in the other half, suggesting a one-tailed test in a particular direction, and guiding the choice of test statistic. The method strongly controls the family-wise error rate, so that the probability that it falsely rejects at least one true null hypothesis is at most $\alpha$ providing the bias in treatment assignment is at most $\Gamma$.

Cross-screening may provide a still stronger conclusion in which the two halves replicate each other, in the sense described by Bogomolov and Heller (2013). Indeed, cross-screening is a modification, at a crucial step, of their replicability method, but refers to random halves of a single study rather than to two independent studies by different investigators. We first define random cross-screening in $\$ 2.1$, discuss aspects of implementation in $\$ 2.2$, and illustrate it in the example in $\$ 2.3$, then discuss its connection to replicability in $\$ 2.4$. Although intuitively pleasing, the technical value of replication with random halves is not obvious; however, in $₫$, we discuss nonrandom cross-screening, and in this case a single investigator may attach importance to replication of two parts of one investigation.

There are $K$ null hypotheses, $H_{k}, k=1, \ldots, K$. In Figure 2, there are $K=46$ null hypotheses, the $k$ th hypothesis asserting no effect of treatment on the $k$ th biomarker. Fix $\alpha$ with $0<\alpha<1$; conventionally, $\alpha=0.05$. If $\mathcal{S}$ is a finite set, write $|\mathcal{S}|$ for the number of elements in $\mathcal{S}$.

A mild premise of our discussion of sample splitting is that the $I$ units in the sample are independent of each other, and that the $K$ hypotheses refer to either a finite or infinite population containing these $I$ units. At the risk of belaboring this premise, it is useful to mention a few particulars to avoid any possibility of misunderstanding. In $\$ 1.3$, there are $I=234$ matched pairs, distinct pairs are assumed independent, and the hypotheses refer either to the $I=234$ pairs as a finite population or to a finite or infinite population from which they were drawn. If the $I$ units are clustered, so that units in distinct clusters are independent, then the clusters, not the $I$ units, should be split at random into two groups of clusters, keeping individual clusters intact in one group or the other. Cross-screening is not applicable if the $I$ units are from a single time series of length $I$. Also, the hypotheses are about the population, whether finite or infinite, in the sense that each $H_{k}$ is either true or false as a description of the population. For example, if $H_{3}$ asserted that at least one person in the finite population of $I=234$ pairs of people is over 100 years old, then $H_{3}$ is true if at least one person in the $I=234$ pairs of people is over 100 years old; otherwise, it is false. When we split the sample in half at random, $H_{3}$ continues to refer to the $I=234$ pairs of people; that is, it does not become two new hypotheses, $H_{3}^{\prime}$ and $H_{3}^{\prime \prime}$, about two randomly defined groups of $234 / 2=117$ pairs of people, where $H_{3}^{\prime}$ might be true and $H_{3}^{\prime \prime}$ 
might be false, depending upon how the random split came out.

Random cross-screening is defined by the following four steps. Essentially, each random half of the data is extensively used to plan the analysis of the other half, and then the two separate analyses are combined by correcting for doing two analyses.

Step 1: Split the sample in half at random.

Step 2: Use the first half in any way at all to select hypotheses $\mathcal{H}_{2} \subseteq\{1, \ldots, K\}$ to test using the second half, with $1 \leq K_{2}=\left|\mathcal{H}_{2}\right| \leq K$. At the same time, use the first half in any way at all to select a test statistic $T_{2 k}$ to use in the second half in testing $H_{k}$ for $k \in \mathcal{H}_{2}$. In the second half, use some method for testing the hypotheses in $\mathcal{H}_{2}$ that would strongly control the family-wise error rate at $\alpha / 2$ if only the $K_{2}$ hypotheses in $\mathcal{H}_{2}$ were tested using $T_{2 k}$ for $k \in \mathcal{H}_{2}$. Let $\mathcal{R}_{2} \subseteq \mathcal{H}_{2}$ be the set of rejected hypotheses.

Step 3: Use the second half in any way at all to select hypotheses $\mathcal{H}_{1} \subseteq\{1, \ldots, K\}$ to test using the first half, with $1 \leq K_{1}=\left|\mathcal{H}_{1}\right| \leq K$. At the same time, use the second half in any way at all to select a test statistic $T_{1 k}$ to use in the second half in testing $H_{k}$ for $k \in \mathcal{H}_{1}$. In the first half, use some method for testing the hypotheses in $\mathcal{H}_{1}$ that would strongly control the family-wise error rate at $\alpha / 2$ if only the $K_{1}$ hypotheses in $\mathcal{H}_{1}$ were tested using $T_{1 k}$ for $k \in \mathcal{H}_{1}$. Let $\mathcal{R}_{1} \subseteq \mathcal{H}_{1}$ be the set of rejected hypotheses.

Step 4: $\quad$ Reject $H_{k}$ if $k \in \mathcal{R}=\mathcal{R}_{1} \cup \mathcal{R}_{2}$.

It is not novel to split a sample at random into two independent subsamples, plan the study using the first subsample, carry out the plan with the independent second subsample, viewing decisions from the first subsample as fixed; see, for instance, Cox (1975) or Heller et al. (2009). That is, Step 2 is not novel on its own, nor is Step 3 on its own. The novel element in cross-screening is that this process is done twice, with each subsample playing both roles, and if either version rejects $H_{k}$ then Step 4 rejects $H_{k}$. In a limited sense, cross-screening uses all of the data to plan and all of the data to test.

The basic property of cross-screening is that it strongly controls the family-wise error rate, as discussed in the following proposition. Strong control of the family-wise error rate means that the probability of falsely rejecting at least one true null hypothesis is at most 
$\alpha$ no matter which of the $K$ hypotheses are true. Let $\mathcal{T} \subseteq\{1, \ldots, K\}$ be the unknown, possibly empty, set of indices such that $H_{k}$ is true if and only if $k \in \mathcal{T}$.

Proposition 1 At least one true null hypothesis, $H_{k}$ with $k \in \mathcal{T}$, is falsely rejected by cross-screening, with $k \in \mathcal{R}$, with probability $\operatorname{Pr}(\mathcal{T} \cap \mathcal{R} \neq \emptyset) \leq \alpha$.

Proof. Write $\mathcal{A}_{1}$ for all of the observed data in the first half sample, and $\mathcal{A}_{2}$ for all of the observed data in the second half sample. From Step 2 of cross-screening, $\operatorname{Pr}\left(\mathcal{T} \cap \mathcal{R}_{2} \neq \emptyset \mid \mathcal{A}_{1}\right) \leq$ $\alpha / 2$, so that $\operatorname{Pr}\left(\mathcal{T} \cap \mathcal{R}_{2} \neq \emptyset\right)=\mathrm{E}\left\{\operatorname{Pr}\left(\mathcal{T} \cap \mathcal{R}_{2} \neq \emptyset \mid \mathcal{A}_{1}\right)\right\} \leq \alpha / 2$. In parallel, from Step $3, \operatorname{Pr}\left(\mathcal{T} \cap \mathcal{R}_{1} \neq \emptyset\right) \leq \alpha / 2$. By the Bonferroni inequality,

$$
\begin{aligned}
& \operatorname{Pr}(\mathcal{T} \cap \mathcal{R} \neq \emptyset)=\operatorname{Pr}\left\{\left(\mathcal{T} \cap \mathcal{R}_{1} \neq \emptyset\right) \text { or }\left(\mathcal{T} \cap \mathcal{R}_{2} \neq \emptyset\right)\right\} \\
& \leq \operatorname{Pr}\left(\mathcal{T} \cap \mathcal{R}_{1} \neq \emptyset\right)+\operatorname{Pr}\left(\mathcal{T} \cap \mathcal{R}_{2} \neq \emptyset\right) \leq \frac{\alpha}{2}+\frac{\alpha}{2}=\alpha .
\end{aligned}
$$

Could use of the Bonferroni inequality in (1) be replaced by an appeal to the independence of the two half-samples, so that $\operatorname{Pr}(\mathcal{T} \cap \mathcal{R} \neq \emptyset)=1-(1-\alpha)^{2}$ instead of $\operatorname{Pr}(\mathcal{T} \cap \mathcal{R} \neq \emptyset) \leq \alpha$ ? It cannot. Although the two half samples are independent of each other, the event $\left(\mathcal{T} \cap \mathcal{R}_{1} \neq \emptyset\right)$ depends upon both halves, as does the event $\left(\mathcal{T} \cap \mathcal{R}_{2} \neq \emptyset\right)$, as seen in Steps 2 and 3 of the description of cross-screening. The choices about which hypotheses to test in the second sample, $\mathcal{H}_{2}$, and the choice of test statistics, $T_{2 k}$, to use when testing hypotheses in $\mathcal{H}_{2}$, were based on the first sample. However, conditionally given those choices, the chance of at least one false rejection in $\mathcal{R}_{2}$ is at most $\alpha / 2$, because the two halves are independent. The situation is parallel for $\mathcal{R}_{1}$.

\subsection{Aspects of implementing cross-screening}

In Step 2 of cross-screening, we would typically uses the first half sample to select a small number $K_{2}=\left|\mathcal{H}_{2}\right|$ of hypotheses, perhaps even $K_{2}=1$, that appear most likely to be rejected when tested in the second half sample. Also, we would pick test statistics, $T_{2 k}$ for $k \in \mathcal{H}_{2}$, that are mostly likely to reject those hypotheses. A simple way to do this is to test every hypothesis $k \in\{1, \ldots, K\}$ using several test statistics in the first half sample, and then pick for use in the second sample the hypotheses and test statistics with the smallest $P$-values in the first sample. Analogous considerations apply to Step 3 of cross-screening. 
In particular, cross-screening permits a few one-sided tests to be performed, rather than many two-sided tests. Shaffer (1974) and Cox $(1977, \S 4.2)$ discuss reasons for viewing a two-sided test of $H_{k}$ as two one-sided tests of $H_{k}$ with a Bonferroni correction for testing $H_{k}$ twice, say by rejecting $H_{k}$ if either $T_{2 k}$ or $-T_{2 k}$ is large. In cross-screening, we might decide in Step 2 to use either $T_{2 k}$ or $-T_{2 k}$, not both, as our test statistic in the second half based on what we observed in the first half, thereby omitting the 2-fold Bonferroni correction needed for a two-sided test. In \$1.3, if the first half sample suggested that eating lots of fish increased a particular biomarker with a long-tailed distribution, we might select that biomarker for testing in the second sample, using a one-sided robust test, looking only for an increase in that biomarker in the second sample.

Suppose that we always picked a single hypothesis to test, so that $1=K_{1}=\left|\mathcal{H}_{1}\right|=$ $K_{2}=\left|\mathcal{H}_{2}\right|$. Then the two halves might select two different hypotheses, $\mathcal{H}_{1} \neq \mathcal{H}_{2}$, and either or both hypotheses might be rejected in Step 4 of cross-screening.

A reasonable strategy is to insist that $K_{1}$ and $K_{2}$ each be at least 1 but much less than $K$. There is little or no hope of outperforming the Bonferroni correction for $K$ hypotheses if $K_{1}$ and $K_{2}$ are near $K$. Even if no hypothesis looks especially promising based on the first half sample, the power of the overall procedure can only be hurt by taking $0=K_{2}=\left|\mathcal{H}_{2}\right|$. In Table 1, we set $K_{1}=K_{2}=2$, selecting the two least sensitive hypotheses in one half-sample for testing in the other half-sample, using the Bonferroni inequality in Steps 2 and 3 to strongly control the family-wise error rate when testing $K_{1}=K_{2}=2$ hypotheses. As it turned out, both half samples selected hypotheses $H_{18}$ and $H_{23}$ for testing in the complementary half; that is, $\mathcal{H}_{1}=\mathcal{H}_{2}=\{18,23\}$. One could instead use Holm's (1979) method for two hypotheses in Steps 2 and 3, thereby gaining in power.

A better approach does not select hypotheses, but rather orders them, and tests them in sequence by a method that controls the family-wise error rate. We recommend ordering hypotheses so that the least sensitive hypothesis appears first. In the planning half, one performs a sensitivity analysis for each outcome, determines the $\Gamma$ at which this outcome becomes sensitive to bias, and orders hypotheses in decreasing order of sensitivity to bias. This approach avoids an arbitrary decision about how many hypotheses to test, but it emphasizes the most promising hypotheses. For methods that control the family-wise error rate at $\alpha$ when testing hypotheses in a given order, see, for instance: Gansky and Koch (1996), Hsu and Berger (1999), Wiens (2003), Hommel and Kropf (2005), Rosenbaum (2008) and Burman, Sonesson and Guilbaud (2009). The simplest approach — so-called 
fixed sequence testing - tests the first hypothesis at level $\alpha$, stops testing if this hypothesis is accepted, otherwise tests the second hypothesis at level $\alpha$, continuing until the first acceptance; see, for instance, Gansky and Koch (1996). Wiens (2003) and Hommel and Kropf (2005) test at a level below $\alpha$, perhaps at $\alpha / 2$, so they can continue beyond the first acceptance; moreover, they transfer unspent $\alpha$ forward to later hypotheses. Wiens calls this a fall-back procedure. Burman et al. (2009) extend that strategy, recycling some unspent $\alpha$ backwards to previously accepted hypotheses. Our simulation in $\$ 5$ evaluates these methods.

The hypotheses $\mathcal{H}_{2}$ and test statistics $T_{2 k}$ picked in Step 2 must be a function of the first half sample, without input from the second half sample. Otherwise, treating $\mathcal{H}_{2}$ and $T_{2 k}$ as fixed when testing in the second sample would not be the same as conditioning on their observed values, a key part of Proposition [1. In parallel, the hypotheses $\mathcal{H}_{1}$ and test statistics $T_{1 k}$ picked in Step 3 must be a function of the second half sample, without input from the first half sample. The most natural, the most convenient, and the most public way to do this is with an explicit function or algorithm that makes these choices, and we recommend that approach in practice. Notice, however, that if, without communicating, one coauthor informally used the first half sample to pick $\mathcal{H}_{2}$ and test statistics $T_{2 k}$, while a second coauthor informally used the second half sample to pick $\mathcal{H}_{1}$ and test statistics $T_{1 k}$, then Proposition 1 would continue to hold.

\subsection{Cross-screening in the example}

Consider blood mercury (LBXTHG or $k=18$ ) in Table1. In a conventional randomization test, $\Gamma=1$, all of the $P$-values for $H_{18}$ are extremely small and both $\mathrm{B}$ and CS reject the hypothesis of no effect. At $\Gamma=9$, the one-sided Wilcoxon test using all of the data has $P$-value bound 0.001036 and multiplying this by $2 \times 46$ yields 0.095 , so method B does not reject in row 18 of in Table 1 .

The illustration of cross-screening in Table 1 selects the $K_{1}=K_{2}=2$ least sensitive hypotheses in each half-sample for testing in the complementary half sample. As it turns out, both half-samples selected hypotheses $H_{18}$ and $H_{23}$ for testing, both referring to mercury levels in the blood. Later, in the simulation in $\$ 5$, we consider methods that do not fix the number of hypotheses to be tested.

In cross-screening, one-sided tests are performed, with the direction of the alternative being determined by the complementary half sample. Additionally, two test statistics were 
tried in one-half sample, and one was selected for testing in the complementary half. One test was Wilcoxon's signed rank test; the other was a U-statistic called $(8,5,8)$ proposed in Rosenbaum (2011) and discussed further in $\S 3$. For hypothesis $H_{18}$, one-sided $P$-value bounds from Wilcoxon's statistic at $\Gamma=9$ are, respectively, 0.03445 and 0.00647 in the first and second half samples. The one-sided $P$-value bounds from the U-statistic $(8,5,8)$ are, respectively, 0.02132 and 0.00383 in the first and second half samples. The quoted $P$-value bound for $k=18, \Gamma=9$ in Table 1 is four times the smallest of these, $0.015=4 \times 0.00383$. This is because, in Step 2, the first sample suggested testing $H_{18}$ and $H_{23}$ in the second sample. Moreover, in Step 2, the first sample suggested using the U-statistic in the second sample because $0.03445>0.02132$. In the second sample, these two hypotheses were tested, and Step 2 requires that the family-wise error in these two tests be controlled at $\alpha / 2$, so each test was performed at level $\alpha / 4=(\alpha / 2) / 2$. The smallest level $\alpha$ that leads to rejection is $0.015=4 \times 0.00383$, yielding the quoted $P$-value from $\mathcal{R}_{2}$. It is beside the point, but nonetheless interesting that the second half sample would also have selected hypotheses $H_{18}$ and $H_{23}$, and would have recommended testing $H_{18}$ with the U-statistic, and it would have yielded a $P$-value bound of $0.0259=4 \times 0.00647$ from $\mathcal{R}_{1}$. So, at $\Gamma=9$, the two halves concur in rejecting $H_{18}$ at the 0.05 level, but we only needed rejection in either half in Step 4. In contrast, at $\Gamma=11$, both half samples recommend testing $H_{18}$ and $H_{23}$ using the U-statistic; however the $P$-value bounds for $H_{18}$ are 0.04589 and 0.00865 in the two halves, so that only the second half sample, $\mathcal{R}_{2}$, leads to rejection with $0.05>0.035=4 \times 0.00865$.

This $P$-value bound of 0.035 from CS should be compared with the $P$-value bound of 0.505 from the Bonferroni method at $\Gamma=11$. The Bonferroni method corrects for $K=46$ two-sided tests, while cross-screening corrects for 4 one-sided tests, two in each of two half samples. Additionally, cross-screening adaptively selected for use in one half sample the test statistic that performed best in the other half sample.

\subsection{Cross-screening and replicability}

With a different goal in view, Bogomolov and Heller (2013) used a procedure similar in form to Steps 1 to 3 of cross-screening, but with a different Step 4, and with some other differences. They asked whether two independent studies by different investigators testing the same $K$ hypotheses have replicated each other. Their method says that rejection of $H_{k}$ has replicated if $k \in \mathcal{R}_{1} \cap \mathcal{R}_{2}$, whereas Step 4 rejects $H_{k}$ in one study if $k \in \mathcal{R}_{1} \cup \mathcal{R}_{2}$; 
that is, both studies must reject $H_{k}$ to replicate, rather than either half rejecting $H_{k}$ to control the family-wise error rate in a single study. Obviously, $\left(\mathcal{R}_{1} \cap \mathcal{R}_{2}\right) \subseteq\left(\mathcal{R}_{1} \cup \mathcal{R}_{2}\right)$ so the probability of replication is lower than the probability of rejection in cross-screening. In the numerical example in 92.3 , hypothesis $k=18 \in \mathcal{R}_{1} \cap \mathcal{R}_{2}$ for $\Gamma=9$, thereby meeting Bogomolov and Heller (2013)'s standard for replication; at $\Gamma=11$, however, $18 \notin \mathcal{R}_{1} \cap \mathcal{R}_{2}$ but $18 \in \mathcal{R}_{1} \cup \mathcal{R}_{2}$, so $H_{18}$ is rejected by cross-screening without meeting the standard for replication.

As Bogomolov and Heller (2013) emphasize, when asking about replicability of two independent studies, it is possible that $H_{k}$ is genuinely true in one study and genuinely false in the other: there might be a treatment effect for outcome $k$ in Cleveland and not in Kyoto, or a specific gene variant might have an effect in a study done on one ethnic group, but not in a study done on a different ethnic group. In contrast, with a random split of a random sample from a single population, $H_{k}$ is either true in the population or false in the population. Additionally, Steps 2 and 3 of cross-screening treat the two half samples symmetrically, each informing the analysis of the other, for instance the choice of test statistic, or the choice of tail for a two-sided test. With two independent studies by different investigators, it is likely that one study came first, so symmetry is unlikely. Perhaps the earlier study influenced the design and analysis of the later study, but the symmetry of Steps 2 and 3 is not expected when one study follows another.

Section 6 discusses an intermediate case involving nonrandom cross-screening in a single study. With nonrandom cross-screening in 6 , it is interesting to know both whether $k \in \mathcal{R}_{1} \cap \mathcal{R}_{2}$ and also whether $k \in \mathcal{R}_{1} \cup \mathcal{R}_{2}$.

\section{Notation for paired randomized experiments and observational studies}

\subsection{Causal inference in paired randomized experiments}

Cross-screening, as described in \$2, is quite general. It could be used with matched pairs, matched sets with multiple controls, full matching, unmatched comparisons, with cohort or case-control studies. The simplest case, however, concerns treatment-control matched pairs, as in 91.3 , and the evaluation in $\$ 4$ will be restricted to this simplest case.

There are $I$ matched pairs, $i=1, \ldots, I$, of two individuals, $j=1,2$, one treated with $Z_{i j}=1$, the other an untreated control with $Z_{i j}=0$, so $1=Z_{i 1}+Z_{i 2}$ for each $i$. Individuals were matched for an observed covariate $x_{i j}$, so that $x_{i 1}=x_{i 2}$ for each $i$, but there is concern about an unmeasured covariate $u_{i j}$ for which it is possible that $u_{i 1} \neq u_{i 2}$ for many or all 
pairs $i$. Write $\mathbf{Z}=\left(Z_{11}, \ldots, Z_{I 2}\right)^{T}$ for the $2 I$ dimensional vector of treatment assignments, and let $\mathcal{Z}$ be the set containing the $2^{I}$ possible values $\mathbf{z}$ of $\mathbf{Z}$, so $\mathbf{z} \in \mathcal{Z}$ if $\mathbf{z}=\left(z_{11}, \ldots, z_{I 2}\right)^{T}$ with $z_{i j}=1$ or 0 and $z_{i 1}+z_{i 2}=1$ for all $i, j$. Conditioning on the event $\mathbf{Z} \in \mathcal{Z}$ is abbreviated as conditioning on $\mathcal{Z}$.

There are $K$ responses, $k=1, \ldots, K$. The $j$ th individual in pair $i$ has two potential outcomes for the $k$ th response, namely $r_{T i j k}$ if this individual is treated with $Z_{i j}=1$ or $r_{C i j k}$ if this individual is given control with $Z_{i j}=0$, so the $k$ th response observed from this individual is $R_{i j k}=Z_{i j} r_{T i j k}+\left(1-Z_{i j}\right) r_{C i j k}$ and the effect caused by the treatment, namely $\delta_{i j k}=r_{T i j k}-r_{C i j k}$, is not observed for any individual; see Neyman (1923) and Rubin (1974). Fisher's (1935) sharp null hypothesis of no treatment effect for response $k$ asserts $H_{k}: \delta_{i j k}=0$ for all $i$ and $j$. Write $\mathbf{R}_{k}=\left(R_{11 k}, \ldots, R_{I 2 k}\right)^{T}$ and $\mathbf{r}_{C k}=\left(r_{C 11 k}, \ldots, r_{C I 2 k}\right)^{T}$, so that $\mathbf{R}_{k}=\mathbf{r}_{C k}$ when $H_{k}$ is true. For the potential outcomes and covariates for the $2 I$ individuals, write $\mathcal{F}=\left[\left\{\left(r_{T i j k}, r_{C i j k}, k=1, \ldots, K\right), x_{i j}, u_{i j}\right\}, i=1, \ldots, I, j=1,2\right]$.

In a paired, randomized experiment, a fair coin is flipped independently $I$ times to assign treatments within the $I$ pairs, so that $\operatorname{Pr}(\mathbf{Z}=\mathbf{z} \mid \mathcal{F}, \mathcal{Z})=2^{-I}=|\mathcal{Z}|^{-1}$ for each $\mathbf{z} \in \mathcal{Z}$. A randomization test of $H_{k}$ compares the distribution of a test statistic, $T_{k}=t_{k}\left(\mathbf{Z}, \mathbf{R}_{k}\right)$, to its randomization distribution, $\operatorname{Pr}\left(T_{k} \geq t \mid \mathcal{F}, \mathcal{Z}\right)$, when $H_{k}$ is true, namely

$$
\operatorname{Pr}\left\{t_{k}\left(\mathbf{Z}, \mathbf{R}_{k}\right) \geq t \mid \mathcal{F}, \mathcal{Z}\right\}=\operatorname{Pr}\left\{t_{k}\left(\mathbf{Z}, \mathbf{r}_{C k}\right) \geq t \mid \mathcal{F}, \mathcal{Z}\right\}=\frac{\left|\left\{\mathbf{z} \in \mathcal{Z}: t_{k}\left(\mathbf{z}, \mathbf{r}_{C k}\right) \geq t\right\}\right|}{|\mathcal{Z}|}
$$

because $\mathbf{R}_{k}=\mathbf{r}_{C k}$ when $H_{k}$ is true, $\mathbf{r}_{C k}$ is fixed by conditioning on $\mathcal{F}$, and $\operatorname{Pr}(\mathbf{Z}=\mathbf{z} \mid \mathcal{F}, \mathcal{Z})=$ $|\mathcal{Z}|^{-1}$ in a randomized experiment.

A common form of test statistic $T_{k}=t_{k}\left(\mathbf{Z}, \mathbf{R}_{k}\right)$ uses the treated-minus-control matchedpair difference, $Y_{i k}=\left(Z_{i 1}-Z_{i 2}\right)\left(R_{i 1 k}-R_{i 2 k}\right)$, which equals $\pm\left(r_{C i 1 k}-r_{C i 2 k}\right)$ when $H_{k}$ is true. The absolute pair differences, $\left|Y_{i k}\right|$, are assigned nonnegative scores $q_{i k} \geq 0$ with $q_{i k}=0$ when $\left|Y_{i k}\right|=0$. Write $\operatorname{sgn}(y)=1$ if $y>0$ and $\operatorname{sgn}(y)=0$ otherwise. The randomization distribution of statistics of the form $T_{k}=\sum_{i=1}^{I} \operatorname{sgn}\left(Y_{i k}\right) q_{i k}$ yield many familiar randomization tests, including: (i) Wilcoxon's signed rank test with $q_{i k}$ to be the rank of $\left|Y_{i k}\right|$, (ii) the permutational $t$-test with $q_{i k}=\left|Y_{i k}\right| / I$, (iii) Maritz (1979)'s randomization distribution for Huber's $M$-statistic with $q_{i k}=\psi\left(\left|Y_{i k}\right| / \varkappa\right)$ where $\varkappa$ is the median $\left|Y_{i k}\right|$ and $\psi(\cdot)$ is a monotone increasing odd function, $\psi(y)=-\psi(-y)$. Under $H_{k}$, the difference $R_{i 1 k}-R_{i 2 k}=r_{C i 1 k}-r_{C i 2 k}$ is fixed by conditioning on $\mathcal{F}$, so the null distribution (2) of $T_{k}$ is the distribution of the sum of $I$ independent random variables taking the values $q_{i k}$ or 0 
with equal probabilities $1 / 2$ if $\left|Y_{i k}\right|>0$ or the value 0 with probability 1 if $\left|Y_{i k}\right|=0$.

The U-statistic $(m, \underline{m}, \bar{m})=(8,5,8)$ was used in cross-screening in Table 1 , Let $a_{i k}$ be the rank of $\left|Y_{i k}\right|$. In general, the U-statistic $(m, \underline{m}, \bar{m})$ has $q_{i k}=\left(\begin{array}{c}I \\ m\end{array}\right)^{-1} \sum_{\ell=\underline{m}}^{\bar{m}}\left(\begin{array}{c}a_{i k}-1 \\ \ell-1\end{array}\right)\left(\begin{array}{c}I-a_{i k} \\ m-\ell\end{array}\right)$; see Rosenbaum (2011). Here, $(m, \underline{m}, \bar{m})=(1,1,1)$ yields the sign test, $(2,2,2)$ is nearly the same as Wilcoxon's test, $(m, \underline{m}, \bar{m})=(m, m, m)$ for integer $m \geq 3$ yields Stephenson's (1981) test, and $(8,5,8)$ is an $S$-shaped transformation of the ranks that diminishes the influence of $Y_{i k}$ that are close to zero.

\subsection{Sensitivity analysis in observational studies}

A simple model for biased treatment assignment in observational studies asserts that in the population, prior to matching, treatment assignments are independent with probabilities $\pi_{i j}=\operatorname{Pr}\left(Z_{i j}=1 \mid \mathcal{F}\right)$, and two subjects, say $i j$ and $i^{\prime} j^{\prime}$, with the same value of $x$, $x_{i j}=x_{i^{\prime} j^{\prime}}$, may differ in their odds of treatment by at most a factor of $\Gamma \geq 1$, that is $\Gamma^{-1} \leq \pi_{i j}\left(1-\pi_{i^{\prime} j^{\prime}}\right) /\left\{\pi_{i^{\prime} j^{\prime}}\left(1-\pi_{i j}\right)\right\} \leq \Gamma$, and then conditions on $\mathbf{Z} \in \mathcal{Z}$. Setting $\gamma=$ $\log (\Gamma) \geq 0$, this is equivalent to introducing an unobserved covariate $\mathbf{u}=\left(u_{11}, \ldots, u_{I 2}\right)^{T}$ with $0 \leq u_{i j} \leq 1$ such that $\operatorname{Pr}(\mathbf{Z}=\mathbf{z} \mid \mathcal{F}, \mathcal{Z})=\exp \left(\gamma \mathbf{u}^{T} \mathbf{z}\right) / \sum_{\mathbf{b} \in \mathcal{Z}} \exp \left(\gamma \mathbf{u}^{T} \mathbf{b}\right)$ for $\mathbf{z} \in \mathcal{Z}$, or equivalently

$$
\operatorname{Pr}(\mathbf{Z}=\mathbf{z} \mid \mathcal{F}, \mathcal{Z})=\prod_{i=1}^{I} \theta_{i}^{z_{i 1}}\left(1-\theta_{i}\right)^{z_{i 2}} \text { with } \frac{1}{1+\Gamma} \leq \theta_{i} \leq \frac{\Gamma}{1+\Gamma} \text { for each } i
$$

see Rosenbaum $(2002, \S 4)$ where equivalences are demonstrated by deriving $u_{i j}$ from $\pi_{i j}$ and conversely. Define $\overline{\bar{T}}_{\Gamma k}$ to be the sum of $I$ independent random variables taking the value $q_{i k} \geq 0$ with probability $\kappa=\Gamma /(1+\Gamma)$ or the value 0 with probability $1 /(1+\Gamma)$, and define $\bar{T}_{\Gamma k}$ analogously but with the probabilities reversed. Then for $T_{k}=\sum_{i=1}^{I} \operatorname{sgn}\left(Y_{i k}\right) q_{i k}$, it is not difficult to show under $H_{k}$ and (3) that the null distribution of $T_{k}$ is bounded by two known distributions,

$$
\operatorname{Pr}\left(\bar{T}_{\Gamma k} \geq t \mid \mathcal{F}, \mathcal{Z}\right) \leq \operatorname{Pr}\left(T_{k} \geq t \mid \mathcal{F}, \mathcal{Z}\right) \leq \operatorname{Pr}\left(\overline{\bar{T}}_{\Gamma k} \geq t \mid \mathcal{F}, \mathcal{Z}\right)
$$

from which bounds on $P$-values, point estimates and confidence intervals are obtained. In particular, the upper bound on the one-sided $P$-value is obtained by evaluating $\operatorname{Pr}\left(\overline{\bar{T}}_{\Gamma k} \geq t \mid \mathcal{F}, \mathcal{Z}\right)$ in (4) with $t$ set to the observed value of the test statistic, $T_{k}$. Under mild conditions on 
the scores, $q_{i k}$, as $I \rightarrow \infty$, the upper bound in (44) can be approximated by

$$
\operatorname{Pr}\left(\overline{\bar{T}}_{\Gamma k} \geq t \mid \mathcal{F}, \mathcal{Z}\right) \doteq 1-\Phi\left(\frac{t-\kappa \sum_{i=1}^{I} q_{i k}}{\sqrt{\kappa(1-\kappa) \sum_{i=1}^{I} q_{i k}^{2}}}\right)
$$

where $\Phi(\cdot)$ is the standard Normal cumulative distribution.

\section{Evaluation of random cross-screening}

\subsection{Random cross-screening performs poorly for $\Gamma=1$ and $K=1$}

Cross-screening can perform poorly. Consider the simplest case, namely one outcome, $K=1$, no bias from unmeasured covariates, $\Gamma=1$, where $Y_{i 1}, i=1, \ldots, I$, are independent observations from a Normal distribution with expectation $\tau$ and variance 1 , $Y_{i 1} \sim_{\text {iid }} N(\tau, 1)$, testing $H_{1}: \tau=0$ against the alternative that $\tau>0 . \quad$ In this case, the uniformly most powerful test is based on the mean, $I^{-1} \sum_{i=1}^{I} Y_{i 1}$, the Bonferroni method uses this best test with no correction because $K=1$, and cross-screening must be inferior. It is a mistake to use cross-screening in a randomized experiment with a small number of null hypotheses.

More generally, we expect random cross-screening to perform poorly in comparison with the Bonferroni-Holm method when there are few hypotheses, $K \leq 10$, and the randomization test, $\Gamma=1$, or tiny biases, $\Gamma=1.1$, are of primary interest.

The situation can be very different when $K$ is large, perhaps $K \geq 100$, and interest is confined to findings that resist a nontrivial bias, say $\Gamma \geq 1.25$. A bias of $\Gamma=1.25$ corresponds with an unobserved covariate $u$ that doubles the odds of treatment, $Z_{i 1}-Z_{i 2}=$ 1 , and doubles the odds of a positive difference in outcomes, $Y_{i k}>0$; see Rosenbaum and Silber (2009b). Reducing $K$ is of substantial value only if $K$ is fairly large.

\subsection{Random cross-screening and design sensitivity}

If there were a treatment effect and no bias from unmeasured covariates in an observational study, then the investigator would not be able to recognize this from the observed data, and the best she could hope to say in this favorable situation is that the conclusions are insensitive to moderate biases as measured by $\Gamma$. In typical situations, as the number of pairs increases, $I \rightarrow \infty$, in this favorable situation, the degree of sensitivity to bias tends to 
a limit, called the design sensitivity, $\widetilde{\Gamma}$, such that the conclusions are eventually insensitive to all biases with $\Gamma<\widetilde{\Gamma}$ and sensitive to some biases with $\Gamma>\widetilde{\Gamma}$; see Rosenbaum (2004; 2010, Part III). More precisely, the upper bound on the $P$-value tends to 1 as $I \rightarrow \infty$ for $\Gamma>\widetilde{\Gamma}$, and it tends to 0 for $\Gamma<\widetilde{\Gamma}$. In that sense, the design sensitivity $\widetilde{\Gamma}$ is the limiting sensitivity to bias as $I \rightarrow \infty$ in a favorable situation. Moreover, the rate at which the $P$-value tends to 0 as $I \rightarrow \infty$ for $\Gamma<\widetilde{\Gamma}$ is the Bahadur efficiency of the sensitivity analysis, and it is useful in characterizing the performance of alternative methods when $\Gamma<\widetilde{\Gamma}$; see Rosenbaum (2015). The Bahadur efficiency drops to zero as $\Gamma$ increases to $\widetilde{\Gamma}$.

Generally, the design sensitivity $\widetilde{\Gamma}$ and Bahadur efficiency depend upon the nature of the favorable situation and on the chosen methods of analysis. A poor choice of test statistic often means an exaggerated report of sensitivity to unmeasured bias, even in large samples. Suppose, for instance, that $Y_{i 1} \sim_{\text {iid }} N(\tau, 1)$, and compare Wilcoxon's signed rank statistic and the U-statistic $(8,5,8)$ that was used in cross-screening in Table 1. If $\tau=1 / 2$, then $\widetilde{\Gamma}=3.2$ for Wilcoxon's statistic, but $\widetilde{\Gamma}=4.2$ for the U-statistic $(8,5,8)$, whereas for $\tau=1$ the design sensitivity is $\widetilde{\Gamma}=11.7$ for Wilcoxon's statistic, but $\widetilde{\Gamma}=26.3$ for the U-statistic; see Rosenbaum (2011, Table 3). At $\tau=1 / 2$, the upper bound on the $P$-value is tending to 1 as $I \rightarrow \infty$ for $\Gamma=3.5>3.2=\widetilde{\Gamma}$ if Wilcoxon's statistic is used, but it is tending to zero if $(8,5,8)$ is used because $\Gamma=3.5<4.2=\widetilde{\Gamma}$. Moreover, with $\tau=1 / 2$ and $\Gamma=2$, the $P$-value bound is tending to zero at a faster rate for $(8,5,8)$ than for Wilcoxon's statistic, with Bahadur relative efficiency $>1.25$; see Rosenbaum (2015, Table 2). A similar pattern is found for a shift of $\tau$ with logistic or $t$-distributed errors. In a randomization test with Normal or logistic errors, the Pitman efficiency of $(8,5,8)$ relative to Wilcoxon's test is 0.97 , so a small loss of efficiency in a randomization test, $\Gamma=1$, translates into substantial gains in a sensitivity analysis, $\Gamma>1$. Although $(8,5,8)$ is consistently slightly better than Wilcoxon's statistic for $\Gamma \geq 2$, other statistics beat $(8,5,8)$ for particular error distributions with no uniform winner; see Rosenbaum (2011, 2015). Adaptive choice of a test statistic can improve design sensitivity and Bahadur efficiency; see Berk and Jones (1978) and Rosenbaum (2012, 2015).

The considerations in the previous paragraph point to one advantage of cross-screening. In Table 1, cross-screening adaptively picked either Wilcoxon's statistic or $(8,5,8)$ based on their performance in the complementary half sample. As $I \rightarrow \infty$ in a favorable situation, if two statistics have different design sensitivities, cross-screening will eventually pick the statistic with the larger design sensitivity and report greater insensitivity to unmeasured bias than would have resulted with a fixed but mistaken choice of statistic. 
The Bonferroni procedure can also be used to adaptively select one of two test statistics, thereby also attaining the better of two design sensitivities. However, the Bonferroni procedure would have to pay for adaptation by testing at level $\alpha /(4 K)$ rather than $\alpha /(2 K)$. There are less costly ways to obtain adaptive inferences; see Rosenbaum (2012).

\subsection{Expected $P$-values and test size in sensitivity analyses with $\Gamma$ slightly too large}

The Bonferroni method in Table 1 made allowance for a 0.05 chance of a false rejection of a true hypothesis in $2 K=96$ one-sided tests. Cross-screening eliminated most hypotheses, focusing on just two of them. Was it wise to do this?

The defining feature of the sensitivity analysis (4) testing $H_{k}$ is that if: (i) $H_{k}$ is true and (ii) the bias in treatment assignment is at most $\Gamma$, then the chance that the $P$-value bound is less than or equal to $\alpha$ is at most $\alpha$ for each $0 \leq \alpha \leq 1$; that is, the $P$-value bound is stochastically larger than the uniform distribution. The Bonferroni method and most if not all methods that use $P$-values in controlling the family-wise error rate are designed so that they give the correct result for $P$-values that are uniformly distributed. However, with $\Gamma>1$, it is common to see many $P$-value bounds that look strictly larger than the uniform distribution.

For example, in the calculations used to carry out the cross-screening in Table 1, a total of $2 \times 2 \times 2 \times 46=368$ one-sided $P$-values were examined, looking at 2 tails, with 2 test statistics, in 2 halves of the data, for 46 outcomes. At $\Gamma=1,40 / 368=11 \%$ of these $P$-values were $\leq 0.05$. However, at $\Gamma=1.25$, only $12 P$-value bounds (4) were $\leq 0.05$, and these occurred for 3 hypotheses $H_{18}, H_{21}$, and $H_{23}$, in both half samples, as judged by both statistics, $12=2 \times 2 \times 3$. Even at $\Gamma=1.25$, small $P$-value bounds were rare, and they may all reflect a genuine effect of eating fish on the level of mercury in the blood.

What happened in Table 1 is not unexpected. Suppose $H_{k}$ is true and the bias in treatment assignment is at most $\Gamma^{\prime}$, but the sensitivity analysis is performed at $\Gamma>\Gamma^{\prime}$. In this case, it is quite unlikely that a true null hypothesis will yield a small $P$-value bound. To demonstrate this, notice that (41) implies the statistic $T_{k}$ is stochastically smaller than $\overline{\bar{T}}_{\Gamma^{\prime} k}$ which is stochastically smaller than $\overline{\bar{T}}_{\Gamma k}$, so $\operatorname{Pr}\left(T_{k} \geq t \mid \mathcal{F}, \mathcal{Z}\right) \leq \operatorname{Pr}\left(\overline{\bar{T}}_{\Gamma^{\prime} k} \geq t \mid \mathcal{F}, \mathcal{Z}\right) \leq$ $\operatorname{Pr}\left(\overline{\bar{T}}_{\Gamma k} \geq t \mid \mathcal{F}, \mathcal{Z}\right)$. Using (5) twice at $\Gamma$ and $\Gamma^{\prime}$, as $I \rightarrow \infty$ we approximate the probability that $\overline{\bar{T}}_{\Gamma^{\prime} k}$ exceeds the upper $\alpha$ critical value for $\overline{\bar{T}}_{\Gamma k}$ as 


$$
1-\Phi\left\{\frac{\left(\kappa-\kappa^{\prime}\right) \sum_{i=1}^{I} q_{i k}+\Phi^{-1}(1-\alpha) \sqrt{\kappa(1-\kappa) \sum_{i=1}^{I} q_{i k}^{2}}}{\sqrt{\kappa^{\prime}\left(1-\kappa^{\prime}\right) \sum_{i=1}^{I} q_{i k}^{2}}}\right\},
$$

where $\kappa^{\prime}=\Gamma^{\prime} /\left(1+\Gamma^{\prime}\right)$. In effect, (6) is an upper bound on the size of an $\alpha$-level sensitivity analysis conducted with the sensitivity parameter $\Gamma$ set above the true bias $\Gamma^{\prime}$. Alternatively, when $H_{k}$ is true, we may approximate the expected $P$-value bound, that is, the expectation of $\operatorname{Pr}\left(\overline{\bar{T}}_{\Gamma k} \geq t \mid \mathcal{F}, \mathcal{Z}\right)$ when $t$ has the distribution of $\overline{\bar{T}}_{\Gamma^{\prime} k}$, as expression (3) in Sackrowitz and Samuel-Cahn (1999),

$$
\mathrm{EPV}=\Phi\left[\frac{\left(\kappa-\kappa^{\prime}\right) \sum_{i=1}^{I} q_{i k}}{\sqrt{\left\{\kappa(1-\kappa)+\kappa^{\prime}\left(1-\kappa^{\prime}\right)\right\} \sum_{i=1}^{I} q_{i k}^{2}}}\right]
$$

see also Dempster and Schatzoff (1965). For Wilcoxon's signed rank statistic without ties, $\sum_{i=1}^{I} q_{i k}=I(I+1) / 2$ and $\sum_{i=1}^{I} q_{i k}^{2}=I(I+1)(2 I+1) / 6$. Both (6) and (7) use the large sample approximation to the distributions of $\overline{\bar{T}}_{\Gamma^{\prime} k}$ and $\overline{\bar{T}}_{\Gamma k}$, so they make no allowance for the discreteness of permutation distributions.

Table 2 evaluates (6) and (7) for a one-sided 0.05-level Wilcoxon test for $I=100,250$ and 500 pairs, for several values of $\Gamma^{\prime} \leq \Gamma$. Of course, when $\Gamma^{\prime}=\Gamma$, the size-bound equals the level-bound, 0.05, and the expected $P$-value is 0.5. However, when $\Gamma^{\prime}<\Gamma$, the size is well below 0.05 and the expected $P$-value is well above 0.5 , a pattern that becomes more noticeable as $I$ increases. If $\Gamma$ is a little too large, small $P$-values for true hypotheses are improbable.

Table 2 is relevant because it is uncommon to see many outcomes that are equally sensitive to unmeasured biases; see, for instance, Table 1. Our tests must allow for this uncommon situation as a logical possibility, but the Bonferroni method and cross-screening do this in two very different ways, though both methods strongly control the family-wise error rate. The Bonferroni method pays a price for every $P$-value bound that is computed. Cross-screening with $\Gamma \geq 1.25$ does not, in typical situations, pursue or spend resources on $P$-value bounds that are very large.

\subsection{Trade-off of sample size $I$ and number of hypotheses $K$ when $\Gamma=1$}

Is it ever worth half the sample to test one hypothesis rather than $K$ hypotheses? Table 3 is several steps removed from cross-screening, but it provides one simple view of the trade- 
off of sample size $I$ and the number of hypotheses under test $K$ when $\Gamma=1$. Consider again the case in which $Y_{i 1} \sim_{\text {iid }} N(\tau, 1)$ and $Y_{i k} \sim_{\text {iid }} N(0,1)$ for $k=2, \ldots, K$, so $H_{1}$ is false and the other $H_{k}$ are true. Table 3 is simply a sample size calculation for the t-test for hypothesis $H_{1}$ in the presence of $K-1$ other true null hypotheses.

Table 3 shows the number of pairs, $I$, required for $80 \%$ power in a single two-sided 0.05-level t-test for $I$ pair differences $Y_{i 1}$ that are $Y_{i 1} \sim_{\text {iid }} N(\tau, 1)$ when a Bonferroni correction is made for testing a total of $K$ hypotheses. For instance, with $\tau=0.1$ and $K=1$ hypothesis one needs $I=787$ pairs for $80 \%$ power, but with $K=50$ hypotheses the Bonferroni correction raises that to 1,713 pairs for $80 \%$ power. In Table 3, sample sizes that are more than twice the sample size for $K=1$ are in bold.

In Table 3, for $K=50,100,250$ and 500 hypotheses, it would be worth discarding a random half of the data if that half correctly identified the one false null hypothesis, thereby eliminated the need to correct for multiple testing in the remaining half of the data. For $K=10$ hypotheses, this is not true, and use of the Bonferroni correction with the full sample yields $80 \%$ power with fewer pairs.

Table 3 is a simple, informal guide, but it is an oversimplification in many ways. First, if several hypotheses were false, not just one, use of the Bonferroni adjustment might reject several hypotheses. Second, cross-screening performs tests in both half-samples, but Table 3 simply discards one half-sample. Third, the conventional t-test has size equal to its level, so the issues in $\$ 4.3$ and Table 2 that arise with $\Gamma>1$ are not reflected in Table 3 .

\subsection{Stylized asymptotics}

One attraction of cross-screening is that it is highly flexible. For instance, one half-sample can adaptively suggest the direction and the test statistic to be used in the other half, and this might be done in a variety of ways. In this section, we greatly restrict, and somewhat distort, the use of cross-screening in the hope of gleaning some analytical insight into its behavior relative to a test that does not split the sample in half. In particular, we do not allow cross-screening to adaptively select a test statistic, a major source of its power. Additionally, we assume that only the first outcome is affected by the treatment and cross-screening selects $K_{1}=K_{2}=1$ hypothesis to test in each half-sample.

Suppose the sample size, $I$, is even and the pairs are split at random in two halves of size $I / 2$. Let $\dot{T}$ and $\ddot{T}$ be the same test statistic computed for the first outcome from the two halves of the data, where we reject $H_{1}$ if the test statistic is large. Because there is 
nothing adaptive here - the choice of test statistic and the direction of the test are fixed in advance - the statistics $\dot{T}$ and $\ddot{T}$ are independent and have the same distribution, whether under the null hypothesis or the alternative. Suppose that the test statistic we would use if we did not split the sample was $\dot{T}+\ddot{T}$. Suppose, finally, that $\dot{T}$ and $\ddot{T}$ would each be $N\left(\theta_{0}, v_{0}\right)$ under the null hypothesis and would each be $N(\theta, v)$ under the alternative. It is convenient to think of $\dot{T}$ and $\ddot{T}$ as scaled so that, as $I \rightarrow \infty$, the expectations, $\theta_{0}$ and $\theta$, are constant, while the variances, $v_{0}$ and $v$, are $O(2 / I)$.

The situation just described would hold exactly with $v=v_{0}$ for the mean of $I / 2$ iid Normally distributed pair differences, and it would hold asymptotically for Wilcoxon's signed rank test and for many other tests for paired data. The signed rank statistic for the whole sample is not quite the same as the sum of the statistics for the two half samples, but as $I \rightarrow \infty$ this distinction would become unimportant. Additionally, $v \neq v_{0}$ for the signed rank statistic.

The combined test, $\dot{T}+\ddot{T}$ is corrected for multiple testing of $K$ hypotheses in 2 tails, so let $\varrho_{b}=2 K$. The combined test rejects the null if $\left(\dot{T}+\ddot{T}-2 \theta_{0}\right) / \sqrt{2 v_{0}} \geq-\Phi^{-1}\left(\alpha / \varrho_{b}\right)$, with power

$\operatorname{Pr}\left\{\frac{\dot{T}+\ddot{T}-2 \theta}{\sqrt{2 v}} \geq \frac{2\left(\theta_{0}-\theta\right)-\Phi^{-1}\left(\alpha / \varrho_{b}\right) \sqrt{2 v_{0}}}{\sqrt{2 v}}\right\}=1-\Phi\left\{\frac{\sqrt{2}\left(\theta_{0}-\theta\right)-\Phi^{-1}\left(\alpha / \varrho_{b}\right) \sqrt{v_{0}}}{\sqrt{v}}\right\}$.

Now, consider cross-screening. Suppose that the first half-sample recommends testing hypothesis $k=1$ in the second half if $\left(\dot{T}-\theta_{0}\right) / \sqrt{v_{0}} \geq-\Phi^{-1}(\alpha)$, and the second half rejects the hypothesis if $\left(\ddot{T}-\theta_{0}\right) / \sqrt{v_{0}} \geq-\Phi^{-1}\left(\alpha / \varrho_{s}\right)$ where $\varrho_{s}=2$ is the cross-screening correction testing for testing one hypothesis in two half samples. In parallel, the second half recommends testing if $\left(\ddot{T}-\theta_{0}\right) / \sqrt{v_{0}} \geq-\Phi^{-1}(\alpha)$ and the first half rejects if $\left(\dot{T}-\theta_{0}\right) / \sqrt{v_{0}} \geq-\Phi^{-1}\left(\alpha / \varrho_{s}\right)$. So cross-screening rejects hypothesis $k=1$ if

$$
\max \left(\frac{\dot{T}-\theta}{\sqrt{v}}, \frac{\ddot{T}-\theta}{\sqrt{v}}\right) \geq \frac{\theta_{0}-\theta-\Phi^{-1}\left(\alpha / \varrho_{s}\right) \sqrt{v_{0}}}{\sqrt{v}}=\lambda_{1}, \text { say, }
$$

and

$$
\min \left(\frac{\dot{T}-\theta}{\sqrt{v}}, \frac{\ddot{T}-\theta}{\sqrt{v}}\right) \geq \frac{\theta_{0}-\theta-\Phi^{-1}(\alpha) \sqrt{v_{0}}}{\sqrt{v}}=\lambda_{2}, \text { say. }
$$


Actually, (9) and (10) embody the following small distortion. In principal, it is logically possible, but not probable, that several of the $K$ hypotheses satisfy (9) and (10). This would complicate the situation; however, it is improbable because only $H_{1}$ is false. For (91) and (10) to hold for a second hypothesis, we would need to reject the same true null hypothesis twice, once in each half-sample.

It is important to notice that $\sqrt{2}\left(\theta_{0}-\theta\right)$ appears on the right of (8), where $\theta_{0}-\theta$ appears in (9) and (10). Therefore, if $I \rightarrow \infty$ with $\varrho_{b}$ and $\varrho_{s}$ fixed - that is, effectively with $K$ fixed - the cross-screening method has little hope of competing with the Bonferroni method. There is, however, a serious competition if $I$ is fixed as $K$ increases, perhaps with $\varrho_{s}=2$ fixed and $\varrho_{b}=2 K$ increasing.

With $\varrho_{s}=2$, we many calculate the probability of the joint event (9) and (10) as

$$
2 \int_{\lambda_{1}}^{\infty} \int_{\lambda_{2}}^{y} \phi(x) \phi(y) d x d y
$$

where $\phi(\cdot)$ is the standard Normal density.

In Table 4, we compare the large-sample power of a nonadaptive cross-screening test of a one-sided hypothesis with a Bonferroni adjusted test of $K$ two-sided hypotheses, where the last $K-1$ null hypotheses are true. The family-wise error rate is controlled at $\alpha=0.05$, so cross-screening tests in two half samples at level $\alpha / 2=0.025$, while the Bonferroni procedure does $2 K$ one-sided tests at level $\alpha /(2 K)$. Here, cross-screening is not permitted to use a major source of its power, namely adaptive testing. Table 4 assumes $v=v_{0}$, as for the Normal-mean situation above, and characterizes power in terms of the noncentrality parameter ncp $=\left(\theta-\theta_{0}\right) / \sqrt{v}$.

In Table 4, we see that cross-screening is a terrible way to select one tail of a two tailed test if there is only $K=1$ hypothesis, that cross-screening is inferior for $K=10$ hypotheses, but that it has higher power than the Bonferroni procedure for $K=100,250$ or 500 hypotheses. Our sense is that Table 4 offers correct qualitative advice: cross-screening is useful when searching for a few large needles in a very big haystack, and is useless in searching for a few hay-like needles in a small haystack. The simulation in \$5 will provide further numerical results about power free of the small distortion noted above. 


\subsection{Splitting with a small planning sample}

An alternative to cross-screening is single screening, as discussed in various contexts by Cox (1975), Heller et al. (2009) and Zhang et al. (2011). In single screening, the sample is split at random into two parts, a small planning sample and a large analysis sample. The study is planned using the planning sample, which is then discarded. For example, one might use the planning sample to order the hypotheses $H_{k}$, determine the side of their one-sided alternatives, and to select test statistics. Then, with the fixed order, sides and test statistics, the analysis sample might test the hypotheses in order. Importantly, split screening discards the planning sample while cross-screening uses it in testing, but split screening plans using a small planning sample while cross-screening uses a half sample.

It only takes a little thought to realize that single screening is better than cross-screening as the sample size grows, $I \rightarrow \infty$, with the number of hypotheses $K$ remaining fixed. See the cited papers for related formal calculations. The reason is that the planning sample only needs to be large enough to ensure that sensible plans are made, so that as $I \rightarrow \infty$ the fraction of the sample needed for planning can diminish and the loss of power from using a planning sample can diminish as well. In an application with $I=132,786$ matched pairs, Zhang et al. (2011) used a 10\% planning sample, where the $10 \%$ reduction in sample size more than paid for itself by improving the plan for analysis of the remaining $90 \%$ of the sample; moreover, $I / 10 \approx 13,800$ pairs was an adequate sample for planning purposes. It is doubtful that results for $I \rightarrow \infty$ with $K$ fixed are relevant to a situation like $\$ 1.3$ with $I=234$ pairs and $K=46$ hypotheses. Ten percent of $I=234$ pairs is only 23 pairs, and that may be too small a planning sample to make correct decisions about $K=46$ hypotheses.

The simulation in $\$ 5$ compares cross-screening to split screening with a $20 \%$ planning sample.

\section{Simulation of a sensitivity analysis testing many null hypotheses}

Table 5 reports simulated power of a level-0.05 sensitivity analysis conducted with $\Gamma=2$ with $K=100$ or $K=500$ hypotheses and $I=100$, or 250 or 500 matched pairs. Among the $K$ hypotheses, one or two are false. These are situations in which cross-screening is expected to perform well: the number of hypotheses is large compared with the sample size, most null hypotheses are true, and a sensitivity analysis is performed with $\Gamma>1$. As noted previously, cross-screening should not be used to test a small number of hypotheses, 
$K$.

There are $K$ independent Normal outcomes with variance 1 . Outcomes $k=1$ and $k=2$ have expectations $\tau_{1}$ and $\tau_{2}$, while $\tau_{k}=0$ for outcomes $k=3, \ldots, K$. That is, hypothesis $H_{1}$ is always false with $\tau_{1} \neq 0, H_{2}$ is false whenever $\tau_{2} \neq 0$, and $H_{k}$ is true for $k=3, \ldots, K$. A value of $K, I$ and $\left(\tau_{1}, \tau_{2}\right)$ defines one sampling situation. In 10,000 replicates of each sampling situation, Table 5 reports the proportion of rejections of $H_{1}$, of $H_{2}$ and of both $H_{1}$ and $H_{2}$. In each sampling situation, the highest power for each hypothesis, $H_{1}, H_{2}, H_{12}$, is in bold.

There are three tests. The first is the familiar Wilcoxon signed rank test. The second test is another signed rank statistic, namely the U-statistic $(8,5,8)$ from Rosenbaum (2011). For many error distributions, the U-statistic $(8,5,8)$ has a larger design sensitivity than Wilcoxon's statistic. Both the Wilcoxon statistic and the U-statistic $(8,5,8)$ are used as fixed tests. The third test makes an adaptive choice between three U-statistics, namely $(8,5,8),(8,6,7)$, and $(8,7,8)$. Of these three, the U-statistic $(8,7,8)$ has the highest design sensitivity for short-tailed distributions like the Normal distribution, $(8,6,7)$ has the highest design sensitivity for long-tailed distributions like the $t$-distribution with 3 degrees of freedom, and $(8,5,8)$ is a compromise; see Rosenbaum (2011, Table 3).

Three methods are used to strongly control the family-wise error rate in two-sided tests. The Bonferroni method splits 0.05 among various hypotheses tests. For instance, using Wilcoxon's test, the Bonferroni method splits 0.05 equally among $K$ tests each with two tails, so a rejection occurs if the one-sided $P$-value bound at $\Gamma=2$ is $\leq 0.05 /(2 K)$. The same approach is used with the U-statistic $(8,5,8)$. For adaptive inference, the Bonferroni method uses all three tests, but rejects if the smallest $P$-value bound (5) is $\leq 0.05 /(3 \times 2 \times K)$. The Bonferroni method attains the largest design sensitivity of the three component tests; however, there are better approaches to adaptive inference (Rosenbaum 2012).

Cross-screening splits the pairs in half at random, plans the analysis in the first half, tests in the second half, then plans in the second half and tests in the first half, with a two-fold Bonferroni correction for having done both analyses. Cross-screening does onetailed tests, not two-tailed tests, having selected one tail based on the planning sample. For adaptive inference, cross-screening picks one of the U-statistics $(8,5,8),(8,6,7)$, and $(8,7,8)$ based on the planning sample. Cross-screening orders the $K$ hypotheses based on the planning sample, tests the hypotheses in the analysis sample in the given order, and stops testing with the first acceptance. It is well known that testing in order strongly 
controls the family-wise error rate; see, for instance, Koch and Gansky (1996), Hsu and Berger (1999), Hommel and Kropf (2005), and Rosenbaum (2008). The hypotheses were ordered by determining their sensitivity to bias in the planning sample, measured by $\Gamma$ at level $\alpha=0.05$, placing the least sensitive hypotheses first.

Single screening split the pairs at random into a $20 \%$ planning sample and an $80 \%$ analysis sample. It discards the $20 \%$ planning sample and does one analysis based on the $80 \%$ analysis sample, without a correction for having done two analyses. In contrast to cross-screening, split screening: (i) omits the correction for having done two analyses, (ii) uses a larger $80 \%$ sample in its one analysis, rather than two $50 \%$ samples, (iii) but discards $20 \%$ of the data. Aside from these differences, the procedures for split screening are the same as for cross-screening. Specifically, in split-screening, the planning sample determines the tail of a one-tailed test, the order for testing-in-order, and in adaptive inference it chooses the test statistic.

In Table 5, the highest power in most sampling situations is from cross-screening with an adaptive choice of test statistic. Wilcoxon's test has inferior power at $\Gamma=2$, consistent with results in Rosenbaum (2011); however, cross-screening often has higher power than the Bonferroni method when the Wilcoxon test is used. The case of $\left(\tau_{1}, \tau_{2}\right)=(0.6,0.4)$ for $I=500$ and $K=100$ is especially interesting. Using adaptive methods, both the Bonferroni method and cross-screening reject $H_{1}$ with power near 1 , but cross-screening has much higher power for $H_{2}$. A similar pattern is seen for $\left(\tau_{1}, \tau_{2}\right)=(0.6,0.4)$ for $I=250$ and $K=100$ and for $I=500$ and $K=500$.

We repeated the simulation in Table 5 but with samples from a t-distribution on 4 degrees of freedom rather than from a Normal distribution. The comparison of Bonferroni, cross-screening and single screening was similar to Table 5, with cross-screening having superior power. Unlike Table 5, adaptive cross-screening was slightly to cross-screening with a fixed choice of the U-statistic $(8,5,8)$, but this is probably because $(8,5,8)$ is an excellent choice for the t-distribution with 4 degrees of freedom; see Rosenbaum (2011, Table 4).

The simulation in Table 5 used screening to order hypotheses which were then tested in a fixed order, terminating with the first acceptance. We also simulated several alternative methods. Wiens (2003), Hommel and Kropf (2005) and Burman et al. (2009) proposed fixed sequence testing procedures that test-in-order at a level below $\alpha$ so that they can continue testing beyond the first acceptance. Wiens (2003) and Hommel and Kropf (2005) transfer forward unspent $\alpha$ to test later hypotheses in the sequence, whereas Burman et 
al. (2009) also cycle back to retest early hypotheses with larger $\alpha$ when later hypotheses are rejected. We tried a so-called fall-back procedure, testing the first hypothesis in order at level $\alpha / 2$, and the second hypothesis at level $\alpha$ if the first hypothesis was rejected or at level $\alpha / 2$ if the first hypothesis was not rejected. We also tried recycling, meaning that if the first hypothesis was not rejected at level $\alpha / 2$, but the second hypothesis was rejected at level $\alpha / 2$, then the first hypothesis was retested at level $\alpha$. In the situations in Table 5 , there are at most two false null hypotheses, so recycling is logically better than fall-back, but this need not be true when three null hypotheses are false. Fixed sequence testing, as reported in Table 5, fall-back and recycling were close competitors for each of the situations in Table 5, and there was no consistent winner for all situations. As logic would suggest, fixed sequence testing had a slight advantage when only $H_{1}$ was false because $\tau_{2}=0$. After all, fixed sequence testing bets all of $\alpha$ on the first hypothesis in the sequence. Similarly, fall-back and recycling had a slight advantage when $\left(\tau_{1}, \tau_{2}\right)=(0.5,0.5)$.

Although we evaluated ordered testing procedures, one need not use any form of ordered testing to use cross-screening. Instead, one could use the first sample to select $K_{1} \ll K$ hypotheses to test in the second sample, and use the second to select $K_{2} \ll K$ hypotheses to test in the second sample, and then correct for multiple testing using Holm's (1979) procedure. As always with cross-screening, a two-fold Bonferroni correction is needed to analyze both half samples.

\section{Nonrandom cross-screening}

In some circumstances, it would be helpful to show that a treatment has the same effect in each of two subpopulations. Nonrandom cross-screening uses an observed binary covariate rather than random numbers to split the sample. Nonrandom and random cross-screening each have advantages and disadvantages.

Suppose that a treatment has a large effect in one subpopulation and no effect in the complementary subpopulation. In this case, nonrandom cross-screening will be unhelpful, because each subpopulation will provide highly misleading advice about how to analyze the complementary subpopulation.

Different people often receive the same treatment for different reasons. One person eats fish because she lives on the coast of Maine and inexpensive fresh fish is abundant, while another person eats fish in Arizona believing it to confer health benefits. When treatments are not randomly assigned, the evidence that the treatment is the cause of its ostensible 
effects is strengthened by showing that people who receive the treatment for different reasons experience similar effects; see Rosenbaum (2001; 2015b, §1.6) With issues of this sort in mind, Lund and Bonaa (1993) examined the possible effects of high fish consumption by comparing the wives of fisherman to controls of similar socioeconomic status. Although the publicly available NHANES data does not classify people by geography or employment, some other data set might permit people who consume high levels of fish to be divided based on whether they eat fish believing it to be health promoting or because of its availability at low cost. In this case, the matched pairs might be divided not at random but to distinguish two reasons people eat fish. The study might be more convincing if it demonstrated the same or similar ostensible effects in both types of pairs.

There is one key technical point about nonrandom cross-screening. If we view a study as testing Fisher's null hypothesis that asserts the treatment has no effect on anyone, then cross-screening may be used as above to test this hypothesis while strongly controlling the family-wise error rate. However, if both splits lead to rejection, then this achieves Bogomolov-Heller replicability, thereby rejecting the null hypothesis of no effect for each subpopulation. With random cross-screening, Bogomolov-Heller replicability does not have a clear interpretation, but with nonrandom cross-screening it constitutes a distinct strengthening of the study's conclusions.

\section{References}

Berk, R. H. and Jones, D. H. (1978), "Relatively optimal combinations of test statistics," Scandinavian Journal of Statistics, 5, 158-162.

Bogomolov, M. and Heller, R. (2013), "Discovering findings that replicate from a primary study of high dimension to a follow-up study," Journal American Statistical Association, 108, 1480-1492.

Burman, C. F., Sonesson, C., and Guilbaud, O. (2009), "A recycling framework for the construction of Bonferroni-based multiple tests," Statistics in Medicine, 28, 739-761.

Cox, D.R. (1975), "A note on data-splitting for the evaluation of significance levels," Biometrika, 62, 441-444.

Cox, D.R. (1977), "The role of significance tests (with Discussion)," Scandinavian Journal of Statistics, 49-70.

Dempster, A. P. and Schatzoff, M. (1965), "Expected significance level as a sensitivity index for test statistics," Journal American Statistical Association, 60, 420-436. 
Fisher, R. A. (1935), Design of Experiments, Ediburgh: Oliver and Boyd.

Fogarty, C. B. and Small, D. S. (2016), "Sensitivity analysis for multiple comparisons in matched observational studies through quadratically constrained linear programming," Journal of the American Statistical Association, 111, 1820-1830.

Heller, R., Rosenbaum, P.R. and Small, D.S. (2009), "Split samples and design sensitivity in observational studies," Journal of the American Statistical Association, 104, 1090-1101.

Holm, S. (1979), "A simple sequentially rejective multiple test procedure," Scandinavian Journal of Statistics," 6, 65-70.

Hommel, G., and Kropf, S. (2005), "Tests for differentiation in gene expression using a data-driven order or weights for hypotheses," Biometrical Journal, 47, 554-562.

Hsu, J. C. and Berger, R. L. (1999), "Stepwise confidence intervals without multiplicity adjustment for dose - response and toxicity studies," Journal of the American Statistical Association, 94, 468-482.

Koch, G. G. and Gansky, S. A. (1996), "Statistical considerations for multiplicity in confirmatory protocols," Drug Information Journal, 30(2), 523-534.

Lund, E. and Bonaa, K. H. (1993), "Reduced breast cancer mortality among fisherman's wives in Norway," Cancer Causes and Control, 4, 283-287.

Neyman, J. (1923, 1990), "On the application of probability theory to agricultural experiments," reprinted in English in: Statistical Science, 5, 463-480.

Rosenbaum, P.R. (1987), "Sensitivity analysis for certain permutation inferences in matched observational studies," Biometrika, 74, 13-26.

Rosenbaum, P. R. (2001), "Replicating effects and biases," American Statistician, 55, 223227.

Rosenbaum, P. R. (2002), Observational Studies ( $2^{\text {nd }}$ edition), New York: Springer.

Rosenbaum, P. R. (2004), "Design sensitivity in observational studies," Biometrika, 91, 153-164.

Rosenbaum, P. R. (2008), "Testing hypotheses in order," Biometrika, 95, 248-252.

Rosenbaum, P. R. (2010), Design of Observational Studies, New York: Springer.

Rosenbaum, P. R. (2011), "A New u-Statistic with superior design sensitivity in matched observational studies," Biometrics, 67, 1017-1027.

Rosenbaum, P. R. (2012), "Testing One Hypothesis Twice in Observational Studies," Biometrika, 99, 763-774.

Rosenbaum, P. R. (2015a), "Bahadur efficiency of sensitivity analyses in observational studies," Journal of the American Statistical Association, 110, 205-217. 
Rosenbaum, P. R. (2015b), "How to see more in observational studies: Some new quasiexperimental devices," Annual Review of Statistics and Its Application, 2, 21-48.

Rosenbaum, P. R. (2016), "Using Scheffé projections for multiple outcomes in an observational study of smoking and periodontal disease," Annals of Applied Statistics, 10, 1447-1471.

Rosenbaum, P.R. and Silber, J.H. (2009a), "Sensitivity analysis for equivalence and difference in an observational study of neonatal intensive care units," Journal of the American Statistical Association, 104, 501-511.

Rosenbaum, P. R. and Silber, J. H. (2009b), "Amplification of sensitivity analysis in observational studies," Journal American Statistical Association, 104, 1398-1405. (amplify function in the $\mathrm{R}$ package sensitivitymv)

Rubin, D. B. (1974), "Estimating causal effects of treatments in randomized and nonrandomized studies," Journal of Educational Psychology, 66, 688-701.

Sackrowitz, H. and Samuel-Cahn, E. (1999), " $P$-values as random variables - expected P-values," American Statistician, 53, 326-331.

Shaffer, J. P. (1974), "Bidirectional unbiased procedures," Journal of the American Statistical Association, 69, 437-439.

Stephenson, W. R. (1981), "A general class of one-sample nonparametric test statistics based on subsamples," Journal of the American Statistical Association, 76, 960-966.

Wiens, B. L. (2003), "A fixed sequence Bonferroni procedure for testing multiple endpoints," Pharmaceutical Statistics, 2, 211-215.

Zhang, K., Small, D. S., Lorch, S., Srinivas, S. and Rosenbaum, P. R. (2011), "Using split samples and evidence factors in an observational study of neonatal outcomes," Journal of the American Statistical Association, 106, 511-524.

Zubizarreta, J. R., Cerdá, M. and Rosenbaum, P. R. (2013), "Effect of the 2010 Chilean earthquake on posttraumatic stress: reducing sensitivity to unmeasured bias through study design," Epidemiology, 24, 79-87. 

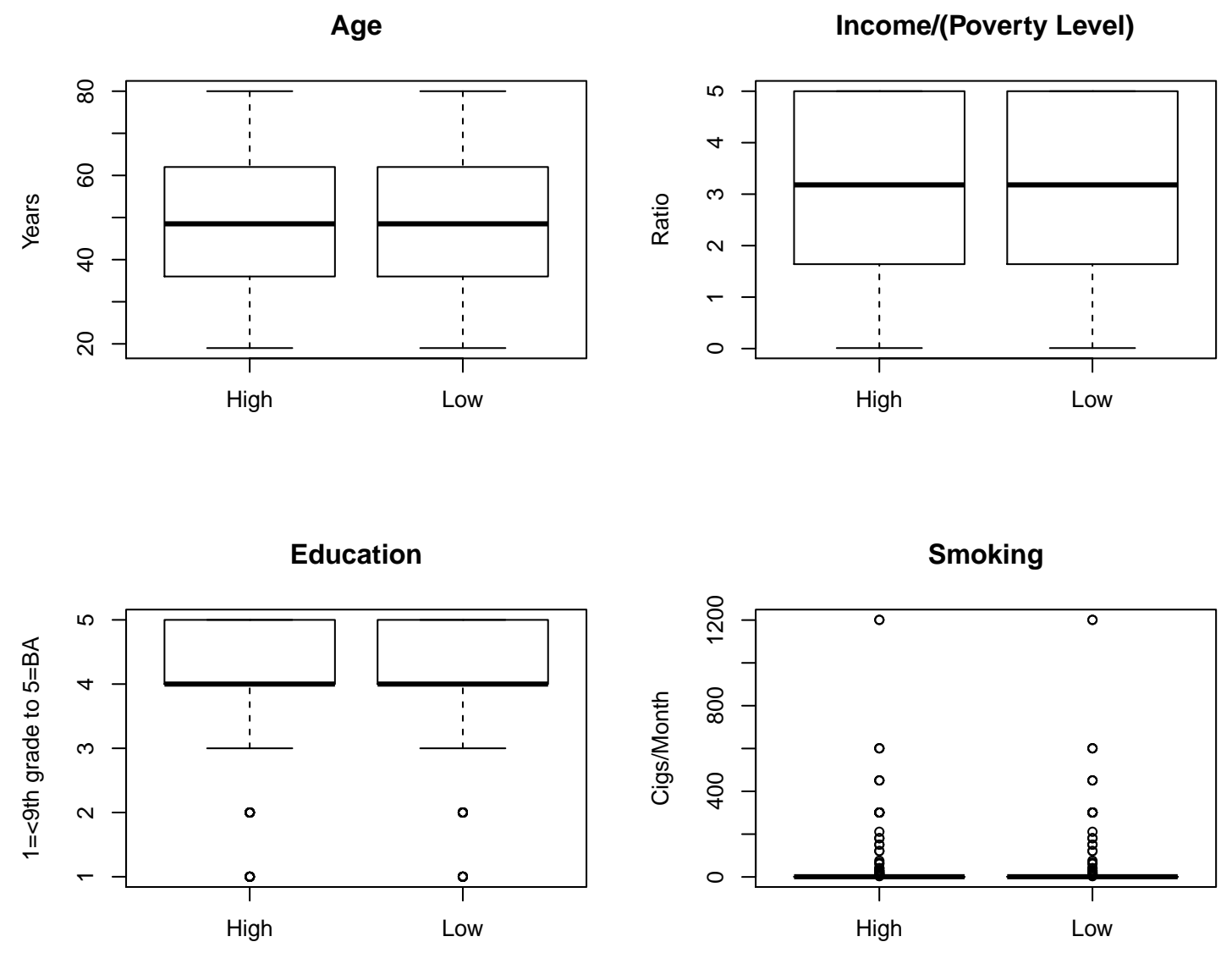

Figure 1: Balance of four covariates in $I=234$ matched pairs of two people, one with a high consump- tion of fish, the other with a low consumption of fish in the past month. 


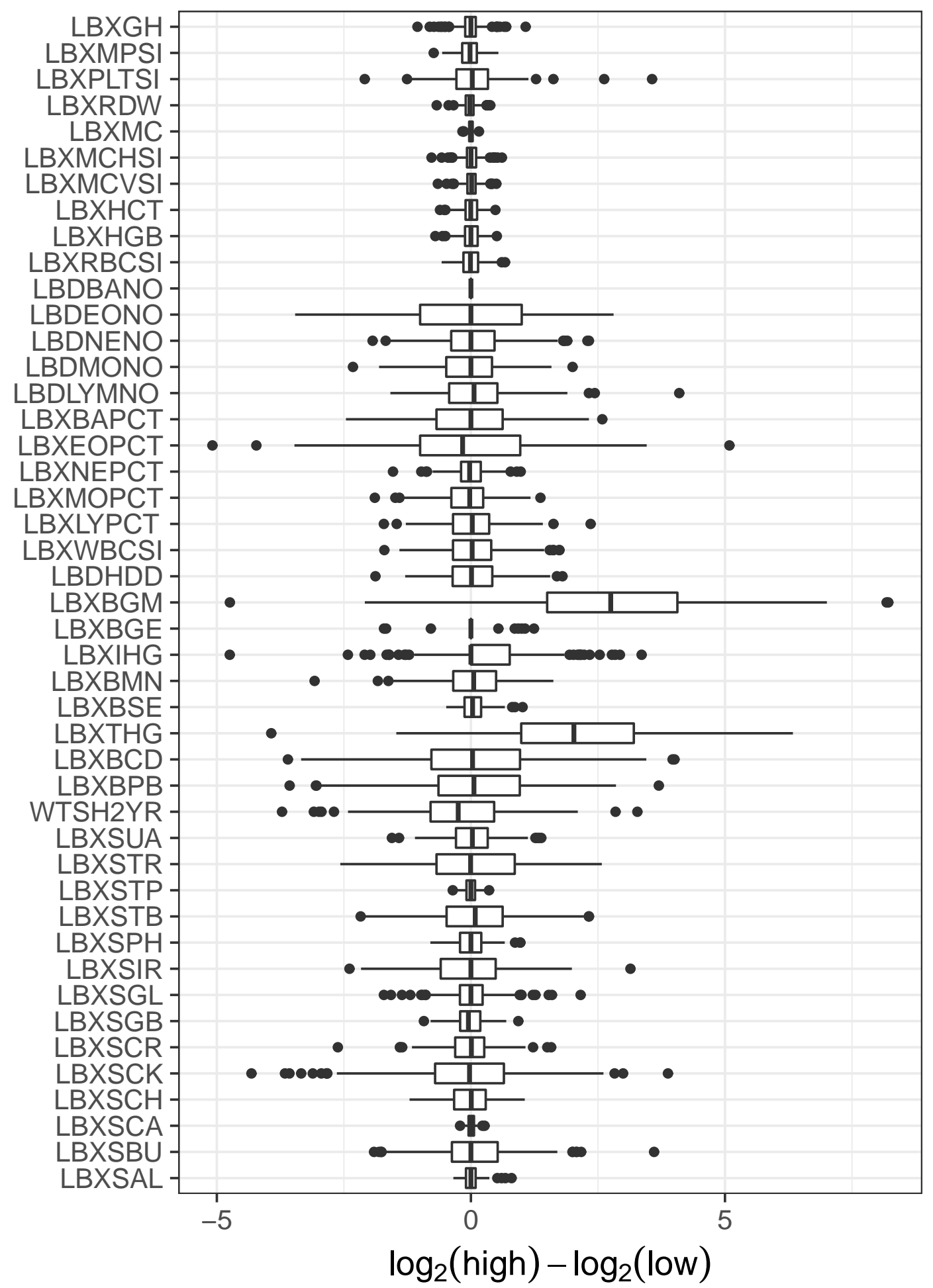

30

Figure 2: $I=234$ High-minus-Low matched pair differences, $Y_{i k}$, of $\log _{2}\left(R_{i j k}\right)$ for outcomes $k=1, \ldots, 46$. A difference of 1 on the $\log _{2}$-scale is 1 doubling. Note the boxplots for LBXBGM and LBXTHG describing mercury in the blood. 
Table 1: Bonferroni correction (B) versus cross-screening (CS) with $K=46$ outcomes and $\Gamma=1,1.25,9$, and 11. A blank indicates that no test was performed.

\begin{tabular}{|c|c|c|c|c|c|c|c|c|c|c|}
\hline \multicolumn{3}{|c|}{ Sensitivity Parameter } & \multicolumn{2}{|c|}{$\Gamma=1$} & \multicolumn{2}{|c|}{$\Gamma=1.25$} & \multicolumn{2}{|c|}{$\Gamma=9$} & \multicolumn{2}{|c|}{$\Gamma=11$} \\
\hline & Outcome & $k$ & $\mathrm{~B}$ & $\mathrm{CS}$ & $\mathrm{B}$ & $\mathrm{CS}$ & $\mathrm{B}$ & $\mathrm{CS}$ & $\mathrm{B}$ & CS \\
\hline LBXSAL & Albumin & 1 & 1.000 & & 1.000 & & 1.000 & & 1.000 & \\
\hline LBXSBU & Blood urea nitrogen & 2 & 1.000 & & 1.000 & & 1.000 & & 1.000 & \\
\hline LBXSCA & Total calcium & 3 & 1.000 & & 1.000 & & 1.000 & & 1.000 & \\
\hline LBXSCH & Cholesterol & 4 & 1.000 & & 1.000 & & 1.000 & & 1.000 & \\
\hline LBXSCK & Creatine phospho. & 5 & 1.000 & & 1.000 & & 1.000 & & 1.000 & \\
\hline LBXSCR & Creatinine & 6 & 1.000 & & 1.000 & & 1.000 & & 1.000 & \\
\hline LBXSGB & Globulin & 7 & 1.000 & & 1.000 & & 1.000 & & 1.000 & \\
\hline LBXSGL & Glucose & 8 & 1.000 & & 1.000 & & 1.000 & & 1.000 & \\
\hline LBXSIR & Iron & 9 & 1.000 & & 1.000 & & 1.000 & & 1.000 & \\
\hline LBXSPH & Phosphorus & 10 & 1.000 & & 1.000 & & 1.000 & & 1.000 & \\
\hline LBXSTB & Total bilirubin & 11 & 1.000 & & 1.000 & & 1.000 & & 1.000 & \\
\hline LBXSTP & Total protein & 12 & 1.000 & & 1.000 & & 1.000 & & 1.000 & \\
\hline LBXSTR & Triglycerides & 13 & 1.000 & & 1.000 & & 1.000 & & 1.000 & \\
\hline LBXSUA & Uric acid & 14 & 1.000 & & 1.000 & & 1.000 & & 1.000 & \\
\hline WTSH2YR & Blood metals & 15 & 0.024 & & 1.000 & & 1.000 & & 1.000 & \\
\hline LBXBPB & Blood lead & 16 & 1.000 & & 1.000 & & 1.000 & & 1.000 & \\
\hline LBXBCD & Blood cadmium & 17 & 1.000 & & 1.000 & & 1.000 & & 1.000 & \\
\hline LBXTHG & Blood mercury & 18 & 0.000 & 0.000 & 0.000 & 0.000 & 0.095 & 0.015 & 0.505 & 0.035 \\
\hline LBXBSE & Blood selenium & 19 & 0.380 & & 1.000 & & 1.000 & & 1.000 & \\
\hline LBXBMN & Blood manganese & 20 & 1.000 & & 1.000 & & 1.000 & & 1.000 & \\
\hline LBXIHG & Mercury, inorganic & 21 & 0.000 & & 0.000 & & 1.000 & & 1.000 & \\
\hline LBXBGE & Mercury, ethyl & 22 & 1.000 & & 1.000 & & 1.000 & & 1.000 & \\
\hline LBXBGM & Mercury, methyl & 23 & 0.000 & 0.000 & 0.000 & 0.000 & 0.075 & 0.014 & 0.405 & 0.031 \\
\hline LBDHDD & HDL-Cholesterol & 24 & 1.000 & & 1.000 & & 1.000 & & 1.000 & \\
\hline LBXWBCSI & White blood cell cnt. & 25 & 1.000 & & 1.000 & & 1.000 & & 1.000 & \\
\hline LBXLYPCT & Lymphocyte \% & 26 & 1.000 & & 1.000 & & 1.000 & & 1.000 & \\
\hline LBXMOPCT & Monocyte $\%$ & 27 & 1.000 & & 1.000 & & 1.000 & & 1.000 & \\
\hline LBXNEPCT & Seg. neutrophils $\%$ & 28 & 1.000 & & 1.000 & & 1.000 & & 1.000 & \\
\hline LBXEOPCT & Eosinophils \% & 29 & 1.000 & & 1.000 & & 1.000 & & 1.000 & \\
\hline LBXBAPCT & Basophils \% & 30 & 1.000 & & 1.000 & & 1.000 & & 1.000 & \\
\hline LBDLYMNO & Lymphocyte \# & 31 & 1.000 & & 1.000 & & 1.000 & & 1.000 & \\
\hline LBDMONO & Monocyte \# & 32 & 1.000 & & 1.000 & & 1.000 & & 1.000 & \\
\hline LBDNENO & Seg. neutrophils \# & 33 & 1.000 & & 1.000 & & 1.000 & & 1.000 & \\
\hline LBDEONO & Eosinophils \# & 34 & 1.000 & & 1.000 & & 1.000 & & 1.000 & \\
\hline LBDBANO & Basophils \# & 35 & 1.000 & & 1.000 & & 1.000 & & 1.000 & \\
\hline LBXRBCSI & Red blood cell cnt. & 36 & 1.000 & & 1.000 & & 1.000 & & 1.000 & \\
\hline LBXHGB & Hemoglobin & 37 & 1.000 & & 1.000 & & 1.000 & & 1.000 & \\
\hline LBXHCT & Hematocrit & 38 & 1.000 & & 1.000 & & 1.000 & & 1.000 & \\
\hline LBXMCVSI & Mean cell volume & 39 & 1.000 & & 1.000 & & 1.000 & & 1.000 & \\
\hline LBXMCHSI & Mean cell hemoglobin & 40 & 1.000 & & 1.000 & & 1.000 & & 1.000 & \\
\hline LBXMC & $\mathrm{MCHC}$ & 41 & 1.000 & & 1.000 & & 1.000 & & 1.000 & \\
\hline LBXRDW & Red cell dist. & 42 & 0.520 & & 1.000 & & 1.000 & & 1.000 & \\
\hline LBXPLTSI & Platelet count & 43 & 1.000 & & 1.000 & & 1.000 & & 1.000 & \\
\hline LBXMPSI & Mean platelet vol. & 44 & $1.00 @ 1$ & & 1.000 & & 1.000 & & 1.000 & \\
\hline LBXGH & Glycohemoglobin & 45 & 1.000 & & 1.000 & & 1.000 & & 1.000 & \\
\hline BPXSY & Systolic BP & 46 & 0.523 & & 1.000 & & 1.000 & & 1.000 & \\
\hline
\end{tabular}


Table 2: When $H_{k}$ is true and the bias in treatment assignment is at most $\Gamma^{\prime}$, the table gives the upper bound on the size of an $\alpha=0.05$ level Wilcoxon test and the upper bound on the expected $P$-value (EPV) if the sensitivity analysis is performed with sensitivity parameter $\Gamma \geq \Gamma^{\prime}$

\begin{tabular}{cc|ccc|ccc}
\hline & & \multicolumn{3}{|c|}{ Bound on Size } & \multicolumn{3}{c}{ Bound on EPV } \\
\hline$\Gamma^{\prime}$ & $\Gamma$ & $I=100$ & $I=250$ & $I=500$ & $I=100$ & $I=250$ & $I=500$ \\
\hline 1.00 & 1.00 & 0.05000 & 0.05000 & 0.05000 & 0.50 & 0.50 & 0.50 \\
1.00 & 1.10 & 0.01976 & 0.01077 & 0.00511 & 0.62 & 0.68 & 0.74 \\
1.00 & 1.25 & 0.00445 & 0.00074 & 0.00007 & 0.75 & 0.86 & 0.94 \\
1.10 & 1.25 & 0.01392 & 0.00586 & 0.00197 & 0.65 & 0.73 & 0.81 \\
1.25 & 1.25 & 0.05000 & 0.05000 & 0.05000 & 0.50 & 0.50 & 0.50 \\
1.25 & 1.50 & 0.00750 & 0.00194 & 0.00033 & 0.71 & 0.81 & 0.89 \\
1.50 & 2.00 & 0.00204 & 0.00017 & 0.00001 & 0.80 & 0.91 & 0.97 \\
\hline
\end{tabular}

Table 3: Number of pairs required for power 0.8 with family-wise error rate 0.05 using the two-sided t-test with a Bonferroni correction for performing $K$ hypothesis tests. When the value in column $K$ is more than twice the value in column $K=1$, the sample size is in bold.

\begin{tabular}{r|rrrrrr}
\hline$\tau$ & $\mathrm{K}=1$ & $\mathrm{~K}=10$ & $\mathrm{~K}=50$ & $\mathrm{~K}=100$ & $\mathrm{~K}=250$ & $\mathrm{~K}=500$ \\
\hline .1 & 787 & 1335 & $\mathbf{1 7 1 3}$ & $\mathbf{1 8 7 4}$ & $\mathbf{2 0 8 7}$ & $\mathbf{2 2 4 7}$ \\
.3 & 89 & 152 & $\mathbf{1 9 5}$ & $\mathbf{2 1 4}$ & $\mathbf{2 3 8}$ & $\mathbf{2 5 6}$ \\
.5 & 33 & 57 & $\mathbf{7 4}$ & $\mathbf{8 1}$ & $\mathbf{9 0}$ & $\mathbf{9 7}$ \\
\hline
\end{tabular}

Table 4: Comparison of the large-sample power of 0.05-level testing of one, one-sided hypothesis by cross-screening (CS) versus testing $K=1,10,50,100,250$ and 500 twosided hypotheses using the Bonferroni method. Cases in which the Bonferroni method has lower power than CS are in bold.

\begin{tabular}{r|r|rrrrrr}
\hline & & \multicolumn{6}{|c}{ Bonferroni with $K$ hypotheses } \\
\hline ncp & CS & 1 & 10 & 50 & 100 & 250 & 500 \\
\hline 1 & 0.0591 & 0.2926 & 0.0818 & $\mathbf{0 . 0 3 0 3}$ & $\mathbf{0 . 0 1 9 4}$ & $\mathbf{0 . 0 1 0 6}$ & $\mathbf{0 . 0 0 6 6}$ \\
2 & 0.3929 & 0.8074 & 0.5085 & $\mathbf{0 . 3 2 2 0}$ & $\mathbf{0 . 2 5 7 1}$ & $\mathbf{0 . 1 8 6 6}$ & $\mathbf{0 . 1 4 4 1}$ \\
3 & 0.8285 & 0.9888 & 0.9244 & 0.8295 & $\mathbf{0 . 7 7 6 9}$ & $\mathbf{0 . 6 9 9 7}$ & $\mathbf{0 . 6 3 7 6}$ \\
\hline
\end{tabular}


Table 5: Simulated power at $\Gamma=2$ with Normal errors for $K$ independent outcomes in $I$ matched pairs. For outcome $k=1$, hypotheses $H_{1}$ is false with pair differences symmetric about $\tau_{1} \neq 0$. For outcome $k=2$, hypotheses $H_{2}$ is false whenever $\tau_{2} \neq 0$. Null hypotheses $k=3, \ldots, K$ are true, with pair differences that are symmetric about zero. Tabulated values are the proportion of rejections at the 0.05 -level in 10,000 replicates. The column labeled $H_{12}$ is the proportion of times both $H_{1}$ and $H_{2}$ were both rejected. In each sampling situation, the highest power for each hypothesis is in bold.

\begin{tabular}{|c|c|c|c|c|c|c|c|c|c|c|c|c|}
\hline & & & & $\mathrm{BO}$ & fferro & & Cros & scree & ing & Sing & e scre & ning \\
\hline$K$ & $I$ & $\left(\tau_{1}, \tau_{2}\right)$ & Statistic & $H_{1}$ & $\mathrm{H}_{2}$ & $H_{12}$ & $H_{1}$ & $\mathrm{H}_{2}$ & $H_{12}$ & $H_{1}$ & $H_{2}$ & $H_{12}$ \\
\hline 100 & 100 & $\overline{(0.5,0.0)}$ & Wilcoxon & 1.4 & & & 23.0 & & & 11.2 & & \\
\hline & & & $(8,5,8)$ & 2.9 & & & 28.4 & & & 13.2 & & \\
\hline & & & Adaptive & 1.3 & & & 27.4 & & & 11.2 & & \\
\hline & & $(0.5,0.5)$ & Wilcoxon & 1.2 & 1.5 & 0.0 & 15.2 & 16.0 & 3.6 & 10.3 & 10.8 & 1.8 \\
\hline & & & $(8,5,8)$ & 3.0 & 2.8 & 0.1 & 19.1 & 19.6 & 5.4 & 11.8 & 11.5 & 2.0 \\
\hline & & & Adaptive & 1.2 & 1.0 & 0.0 & 21.3 & 21.6 & 6.7 & 10.9 & 11.4 & 1.8 \\
\hline & & $(0.6,0.4)$ & Wilcoxon & 9.6 & 0.2 & 0.0 & 47.8 & 3.7 & 2.6 & 28.8 & 2.5 & 1.1 \\
\hline & & & $(8,5,8)$ & 14.5 & 0.2 & 0.0 & 52.1 & 5.0 & 3.5 & 28.5 & 3.0 & 1.1 \\
\hline & & & Adaptive & 7.8 & 0.1 & 0.0 & 50.9 & 6.1 & 4.2 & 25.5 & 3.7 & 1.3 \\
\hline & 250 & $(0.5,0.0)$ & Wilcoxon & 18.7 & & & 72.7 & & & 57.8 & & \\
\hline & & & $(8,5,8)$ & 31.1 & & & 82.1 & & & 60.9 & & \\
\hline & & & Adaptive & 35.0 & & & 88.3 & & & 60.0 & & \\
\hline & & $(0.5,0.5)$ & Wilcoxon & 19.4 & 17.9 & 3.5 & 53.7 & 53.1 & 39.8 & 54.0 & 53.3 & 34.0 \\
\hline & & & $(8,5,8)$ & 30.5 & 30.5 & 9.5 & 66.7 & 67.1 & 56.3 & 59.0 & 59.2 & 39.1 \\
\hline & & & Adaptive & 36.3 & 35.1 & 12.6 & 79.3 & 79.2 & 70.7 & 59.5 & 59.3 & 38.9 \\
\hline & & $(0.6,0.4)$ & Wilcoxon & 71.4 & 0.9 & 0.6 & 90.4 & 20.7 & 20.6 & 78.5 & 15.3 & 13.7 \\
\hline & & & $(8,5,8)$ & 83.0 & 2.8 & 2.3 & 93.6 & 31.8 & 31.7 & 79.9 & 21.4 & 19.3 \\
\hline & & & Adaptive & 84.2 & 4.2 & 3.5 & 95.5 & 44.4 & 44.2 & 78.4 & 25.5 & 22.2 \\
\hline & 500 & $(0.5,0.0)$ & Wilcoxon & 66.7 & & & 96.3 & & & 95.3 & & \\
\hline & & & $(8,5,8)$ & 82.8 & & & 98.8 & & & 95.9 & & \\
\hline & & & Adaptive & 92.7 & & & 99.8 & & & 95.0 & & \\
\hline & & $(0.5,0.5)$ & Wilcoxon & 66.8 & 66.5 & 44.6 & 91.3 & 91.1 & 88.2 & 93.8 & 93.9 & 90.1 \\
\hline & & & $(8,5,8)$ & 82.6 & 82.7 & 68.6 & 96.5 & 96.8 & 95.7 & 95.7 & 95.9 & 92.6 \\
\hline & & & Adaptive & 92.7 & 92.7 & 85.9 & 99.4 & 99.5 & 99.2 & 95.4 & 95.1 & 91.2 \\
\hline & & $(0.6,0.4)$ & Wilcoxon & 99.3 & 4.8 & 4.8 & 99.4 & 45.9 & 45.9 & 95.5 & 46.1 & 46.0 \\
\hline & & & $(8,5,8)$ & 99.9 & 13.1 & 13.1 & 99.8 & 64.9 & 64.9 & 97.0 & 60.7 & 60.5 \\
\hline & & & Adaptive & 100.0 & 30.8 & 30.8 & 100.0 & 86.5 & 86.5 & 98.4 & 70.8 & 70.6 \\
\hline 500 & 500 & $(0.5,0.0)$ & Wilcoxon & 47.5 & & & 96.3 & & & 90.5 & & \\
\hline & & & $(8,5,8)$ & 68.4 & & & 99.0 & & & 90.7 & & \\
\hline & & & Adaptive & 83.0 & & & 99.8 & & & 87.8 & & \\
\hline & & $(0.5,0.5)$ & Wilcoxon & 48.5 & 48.8 & 23.7 & 91.1 & 91.2 & 88.3 & 89.4 & 89.5 & 82.0 \\
\hline & & & $(8,5,8)$ & 68.7 & 69.0 & 47.7 & 96.8 & 96.7 & 95.7 & 90.3 & 90.6 & 82.9 \\
\hline & & & Adaptive & 83.7 & 83.9 & 70.3 & 99.5 & 99.5 & 99.3 & 88.2 & 88.7 & 79.1 \\
\hline & & $(0.6,0.4)$ & Wilcoxon & 97.8 & 1.8 & 1.7 & 99.5 & 45.9 & 45.9 & 94.7 & 39.0 & 38.6 \\
\hline & & & $(8,5,8)$ & 99.3 & 5.8 & 5.7 & 99.7 & 63.9 & 63.9 & 96.2 & 49.5 & 49.0 \\
\hline & & & Adaptive & 99.9 & 16.2 & 16.2 & 100.0 & 85.7 & 85.7 & 96.9 & 57.2 & 56.2 \\
\hline
\end{tabular}

